\title{
Designing Selective and Non-Immunosuppressive Antifungal FK506 Analogs: Structures, Biophysics and Dynamics of Fungal and Human Calcineurin-Inhibitor Complexes
}

Sophie M.-C. Gobeil ${ }^{1,2}$, Benjamin G. Bobay ${ }^{3}$, Praveen R. Juvvadi ${ }^{4}$, D. Christopher Cole ${ }^{4}$, Joseph Heitman $^{5}$, William J. Steinbach ${ }^{4,5}$, Ronald A. Venters ${ }^{3}$, and Leonard D. Spicer ${ }^{1,2,3, \dagger}$.

\author{
Affiliations \\ ${ }^{1}$ Department of Biochemistry, Duke University, Durham, NC 27710, USA. \\ ${ }^{2}$ Department of Radiology, Duke University, Durham, NC 27710, USA. \\ ${ }^{3}$ Duke University NMR Center, Duke University Medical Center, Durham, NC 27710, USA. \\ ${ }^{4}$ Division of Pediatric Infectious Diseases, Department of Pediatrics, Duke University Medical \\ Center, Durham, NC 27710, USA. \\ 5 \\ 5 Department of Molecular Genetics and Microbiology, Duke University Medical Center, \\ Durham, NC 27710, USA. \\ ${ }^{\dagger}$ Address correspondence to Leonard D. Spicer, spicer@biochem.duke.edu
}




\begin{abstract}
Calcineurin is an attractive antifungal target due to its central role in fungal pathogenesis. The development of specific antifungals targeting calcineurin is complex, as calcineurin inhibitors, such as FK506, are immunosuppressive. Using fungal calcineurin-inhibitor crystal structures we recently developed a less immunosuppressive FK506 analog, APX879, with broad-spectrum antifungal activity and efficacy in a murine model of invasive fungal infection. To better understand the interaction of the human and fungal FK506-binding proteins (FKBP12) required for calcineurin inhibition at a molecular level, and guide the design of fungal-selective and nonimmunosuppressive FK506 analogs, here we report the high-resolution structures of the $M$. circinelloides FKBP12 bound to FK506 and the human, A. fumigatus and M. circinelloides FKBP12 proteins bound to the FK506 analog, APX879. Combining structural, genetic and biophysical methodologies with molecular dynamics simulations, we identify critical variations in these structurally similar FKBP12:ligand complexes to enhance fungal-selectivity.
\end{abstract}

\title{
Keywords
}

FK506, calcineurin, FKBP12, APX879, Antifungals, M. circinelloides, A. fumigatus, NMR, Xray crystal structures, isothermal titration calorimetry (ITC), molecular dynamic (MD) simulations 
Invasive fungal infections are a leading cause of death in immunocompromised patients. More than 1.6 million people die annually of infections caused by the major fungal pathogenic species of Aspergillus, Candida, Cryptococcus, and Mucorales ${ }^{1}$. Due to rapidly emerging drug resistance to the existing arsenal of antifungals targeting the fungal cell wall and membrane, there is an urgent need to design more efficacious and highly selective antifungal drugs targeting other critical fungal cellular pathways. However, this poses a fundamental challenge as both fungi and humans are eukaryotes and share many orthologous proteins and pathways ${ }^{2}$.

Calcineurin, the target of the immunosuppressive macrocyclic drugs FK506 (tacrolimus) and cyclosporin A (CsA), is a promising target for the development of effective antifungal drugs ${ }^{3}$. Calcineurin plays central roles in fungal growth, pathogenesis, cellular stress responses, and drug resistance ${ }^{4}$. The calcineurin protein complex consists of a catalytic subunit, calcineurin $\mathrm{A}(\mathrm{CnA})$, and a regulatory subunit, calcineurin $\mathrm{B}(\mathrm{CnB})^{5}$. The immunosuppressants first bind to their respective immunophilins, FKBP12 (12-kDa FK506 binding protein) and CypA (cyclophilin A), which subsequently bind to calcineurin in a groove between the $\mathrm{CnA}$ and $\mathrm{CnB}$ subunits. The immunophilin-immunosuppressant complex inhibits calcineurin serine-threonine phosphatase activity blocking the dephosphorylation of downstream targets, such as the human nuclear transcription factor NFAT involved in T-cell activation and interleukin-2 transcription, and the fungal transcription factor Crz1 (NFAT ortholog), implicated in virulence, stress response, and thermotolerance ${ }^{6-10}$. In humans, this leads to potent immunosuppression and is critical in preventing graft rejection, but also precludes FK506 and CsA usage as antifungals in already immunocompromised patients.

FKBP12 proteins are members of the FKBP PPIase (peptidylprolyl isomerase) superfamily and catalyze the cis-trans isomerization of proline imidic peptide bonds ${ }^{11-18}$. Based on our recent 
characterization of the FK506-FKBP12 and calcineurin-FK506-FKBP12 crystal structures $^{19,20}$, we synthesized an FK506 analog, APX879, modified with an acetohydrazine moiety on FK506C22 (Fig. 1A). We proposed that APX879 would interact less favorably with the human FKBP12 (hFKBP12) His88 residue than with the corresponding A. fumigatus FKBP12 (AfFKBP12) Phe88 residue thus, potentially reducing immunosuppression ${ }^{20}$. APX879 displayed a 71-fold reduced immunosuppressive activity compared to FK506 while maintaining broadspectrum in vitro antifungal activity against a wide range of human pathogenic fungi ${ }^{20}$. In vivo testing in murine model confirmed reduced immunosuppressive activity, and efficacy in a Cryptococcal model of invasive fungal infection ${ }^{20}$.

To guide the design of non-immunosuppressive FK506 analogs selectively targeting fungal calcineurin, here, we quantitatively compare FK506 and APX879 binding to the human and fungal FKBP12 proteins from A. fumigatus and $M$. circinelloides through a combination of genetic, structural, and biophysical approaches. As $M$. circinelloides is an emerging human pathogen recalcitrant to the majority of current antifungals, molecular characterization of the FKBP12 protein $\left(M c\right.$ FKBP12) is of high interest to enable efficient targeting of calcineurin ${ }^{21,22}$. Here, we report the first high-resolution crystal structures of McFKBP12 bound to FK506 in addition to the $h \mathrm{FKBP} 12$, AfFKBP12, and $M c$ FKBP12 proteins bound to APX879 ${ }^{20}$. Through genetic studies, we demonstrate that $M c$ FKBP12 does not functionally complement AfFKBP12 and reveal key requirements of FKBP12 residue 88 for productive binding and inhibition of calcineurin. While FK506 binds to both human and fungal FKBP12 proteins with high affinity (2-5 nM), APX879 binds 40- to 100-fold less tightly (120-450 nM). Strikingly, the human and fungal FKBP12 proteins show different responses to APX879 binding as observed by NMR, isothermal calorimetric titration (ITC) experiments, and molecular dynamic (MD) simulations 
that are not readily apparent in their static X-ray crystal structures. Furthermore, MD simulations allowed quantitative comparison of the significance of the FKBP12:ligand interactions between the human and fungal proteins. This analysis reveals regions of the ligands that could be altered to enhance selectivity toward the fungal FKBP12 proteins. Our approach highlights the potential of combining structural, genetic, biophysical, and in silico methodologies to fully describe and identify variation in protein:ligand interactions involving structurally similar proteins. We take a rational approach to understand the balance between the immunosuppressive and antifungal activities of FK506 and a new, less immunosuppressive analog, APX879, to broaden the therapeutic window for the development of efficacious antifungals.

\section{Results}

Crystal structure of M. circinelloides FKBP12 bound to FK506

$M c \mathrm{FKBP} 12$ shares $58 \%$ and $65 \%$ sequence identity with $h \mathrm{FKBP} 12$ and $A f \mathrm{FKBP} 12$, respectively. Sequence variations to $h \mathrm{FKBP} 12$ are located in: (i) $\beta 2$, a back wall of the FK506binding pocket leading into the 40's loop, (ii) the 50's loop, and (iii) $\beta 4$, leading into the 80's loop (Figs. 1B and 2A). Crystal structures of the apo and FK506-bound $h$ FKBP12 and AfFKBP12 proteins have been reported ${ }^{19,23,24}$. To correlate sequence variations with structure and identify potential differences between $M c$ FKBP12 and other FKBP12 proteins, we attempted to crystallize $M c$ FKBP12 in its apo form. All attempts failed to yield protein crystals hinting at the potentially high conformational flexibility of $M c$ FKBP12. However, crystals were obtained with FK506 (2.5 ̊, P32 21$)$ suggesting rigidification of $M c$ FKBP12 by FK506 binding (Supplementary Table 1). 
$M c$ FKBP12 shares the same fold as $h \mathrm{FKBP} 12$ and $A f \mathrm{FKBP} 12(\mathrm{C} \alpha-\mathrm{RMSD} \sim 0.5 \AA$ ) with a 5-stranded $\beta$-sheet wrapping around an $\alpha$-helix (Fig. 2A). The three main loops defining the FK506-binding cavity (40's, 50's, and 80's loops) adopt the same conformation as observed in apo and FK506-bound forms of other FKBP12 proteins. When bound to McFKBP12, FK506 also adopts the same conformation as when bound to other mammalian and fungal FKBP12 proteins (FK506 RMSD $\sim 0.3 \AA$ A). Five hydrogen bonds (H-bonds) between FK506 and McFKBP12 are observed: 2 involving the backbone of residues Gln55 and Ile57, and 3 to the side chains of residues Tyr27, Asp38, and Tyr83 (Fig. 2B). Despite the sequence differences between $M c$ FKBP12 and AfFKBP12, they share high structural similarity, prompting investigation into their functional equivalence.

\section{M. circinelloides FKBP12 does not functionally complement A. fumigatus FKBP12 in}

\section{calcineurin inhibition}

The deletion of A. fumigatus AfFKBP12 encoding gene leads to FK506 resistance, establishing its central role for calcineurin inhibition ${ }^{25}$. To verify if $M c$ FKBP12 can functionally complement AfFKBP12, an A. fumigatus strain expressing $M c$ FKBP12 was generated through genetic replacement of the Affkbp12 native locus with Mcfkbp12 (Supplementary Fig. 1). Growth assays in the presence of increasing concentrations of FK506 revealed that McFKBP12 does not restore $A$. fumigatus FK506 sensitivity indicating that $M c$ FKBP12 may not bind or may bind but not inhibit A. fumigatus calcineurin in vivo (Fig. 3A and Supplementary Fig. 2), consistent with previous analysis using $h \mathrm{FKBP} 12^{20}$. Using structure and sequence alignments, a single mutation of $h \mathrm{FKBP} 12-\mathrm{His} 88$ to Phe (AfFKBP12 identity), was shown to restore FK506 sensitivity $^{20}$. Interestingly, in $M c$ FKBP12 residue 88 is a Tyr, potentially sterically hindering the ternary complex formation with A. fumigatus calcineurin. To test this hypothesis, we generated a 
McFKBP12-Y88F mutant and confirmed restoration of FK506 sensitivity in A. fumigatus (Fig. 3A, Supplementary Figs. 1 and 2). We also verified the calcineurin-FK506-FKBP12 complex formation in vivo using GFP tagged $M c$ FKBP12 and $M c$ FKBP12-Y88F expression constructs by fluorescence microscopy (Fig. 3B and Supplementary Fig. 1). In the absence of FK506, as observed with AfFKBP12, the $M c$ FKBP12 and $M c$ FKBP12-Y88F proteins localize in the cytoplasm and nuclei. Upon FK506 addition, both proteins localize to the septum as observed with $A f \mathrm{FKBP} 12$, demonstrating the formation of the ternary complex with calcineurin at the hyphal septum, independent of the Y88F mutation. This highlights the central role of residue 88 in the formation of a "productive" inhibitory complex with A. fumigatus calcineurin. Despite the binding of $M c \mathrm{FKBP} 12$ (and $h \mathrm{FKBP} 12$ ) to A. fumigatus calcineurin in vivo in the presence of FK506, subsequent inhibition is dependent on the presence of the critical Phe residue at position 88 suggesting that the side chain size ( $M c$ FKBP12-Tyr88) and charge ( $h$ FKBP12-His88) control the formation of a "productive" inhibitory protein-protein interface.

To assess the structural implications for FK506 binding of the Tyr88Phe substitution, we performed 500 ns $\mathrm{MD}$ simulations on $M c \mathrm{FKBP} 12, \quad M c \mathrm{FKBP} 12-\mathrm{Y} 88 \mathrm{~F}$, and AfFKBP12 (Supplementary Fig. 3). While these data suggest that the Y88F mutation in McFKBP12 does not grossly alter the conformational state, $\mathrm{C} \alpha-\mathrm{RMSF}$ of $M c \mathrm{FKBP} 12$, or average number of $\mathrm{H}-$ bonds between FKBP12 and FK506 (McFKBP12: 1.5, McFKBP12-Y88F: 1.7 and AfFKBP12: 2.1), it does alter which residues of $M c \mathrm{FKBP} 12$ and atoms of FK506 are forming H-bonds pairs.

H-bonds to FK506 involving residues 27, 38, 43, 54, 55, 57, 60, 83, 85, 86-88, and 91 are observed, at one point or another, for $M c \mathrm{FKBP} 12, M c \mathrm{FKBP} 12-\mathrm{Y} 88 \mathrm{~F}$, and $A f \mathrm{FKBP} 12$ during the simulations confirming the central role of the 50's and 80's loops in FK506 binding and correlating with the crystal structures (Fig. 3C). Interestingly, the H-bonds between FK506 and 
McFKBP12-Y88F residues Ser39-OG, Thr50-OG1, Gly54-N, Val56-O, and Tyr83-OH are disrupted in comparison to McFKBP12 while new H-bonds involving Lys58, Tyr83, Gly84, and Glu85 backbone are observed. These latter two new H-bonds, implicating the 80's loop, are also observed for AfFKBP12-FK506. This suggests that the Y88F mutation shifts the significance of the 50's and 80's loop in the interaction of McFKBP12 with FK506. These results emphasize the central role of AfFKBP12-Phe88 for the formation of a productive FK506:FKBP12 composite surface allowing for calcineurin inhibition.

\section{Crystal structures of the human and fungal FKBP12 proteins bound to APX879}

The FK506 analog, APX879 substituted on the C22-ketone with an acetohydrazine moiety, was hypothesized to introduce a steric clash with $h$ FKBP12-His88 benefiting fungalselectivity (Fig. 1A). ${ }^{20}$ To understand the differential binding of APX879 to the fungal and human FKBP12 proteins, we obtained each of these X-ray crystal structures (Fig. 4 and Supplementary table 1). Crystals of $h \mathrm{FKBP} 12$, AfFKBP12, and $M c$ FKBP12 bound to APX879 diffracted at resolutions of 1.7, 1.6, and $1.9 \AA$ respectively. The APX879-FKBP12 structures showed minimal structural variations compared to their FK506-bound counterparts (C $\alpha$-RMSDs $\sim 0.5 \AA$ ) maintaining similar H-bonds pattern (i.e. 4 to 5): two with the backbone of residues $\mathrm{Glu}_{h} / \operatorname{Arg}_{A f} / \mathrm{Gln}_{M c} 55$ and Ile57 and two/three with the side chain of residues Tyr27, Asp38, and Tyr83.

Previous crystal structures of AfFKBP12 bound to FK506 required a Pro90Gly mutation to trap the ligand in the binding cavity as without this mutation, an apo homodimer hypothesized to result from a self-catalysis mechanism involving the Pro89-Pro90 motif, was captured ${ }^{19}$. Here, we obtained crystals of AfFKBP12 bound to APX879 without the requirement for a P90G mutation by the addition of APX879 during purification. Interestingly, Pro90 adopted the cis 
conformation, as observed in the crystal structure of the calcineurin-FK506-FKBP12 complex $(\mathrm{PDB} 6 \mathrm{TZ7})^{20}$. In contrast, the human and $M c$ FKBP12 proteins, having a Pro89-Gly90 motif, do not show any evidence of an intermolecular self-catalysis-like binding event. The Pro90 cis conformer in AfFKBP12 allows the 80's loop to reduce the size of the FK506-binding pocket as estimated by $3 \mathrm{~V}$ volume calculations (PDB 5HWB; trans conformer $\sim 390 \AA^{3}$; cis conformer 240 $\AA^{3}$ ) to a volume similar to other FKBP12 proteins with a Gly90 residue ( $h$ FKBP12 $220 \AA^{3}$, PDB 2PPN) ${ }^{26}$. Using apo and FK506-bound AfFKBP12 NMR assignments we also confirmed the adoption of the Pro90 cis conformer in solution as suggested by the $\mathrm{C} \beta$ (apo: 33.2 ppm; FK506bound: $34.2 \mathrm{ppm}$ ) and $\mathrm{C} \gamma$ (apo: $24.2 \mathrm{ppm}$ ) resonances ${ }^{27-29}$. Our AfFKBP12-APX879 crystal structure further emphasizes that Pro90 cis conformation might modulate the binding cavity volume.

We next assessed APX879 acetohydrazine moiety accommodation by the different FKBP12 proteins. Similar to the $h$ FKBP12-FK506 structure, in the $h$ FKBP12-APX879 crystal structure a $\mathrm{C} 21-\mathrm{C} 22$ dihedral angle of $36^{\circ}$ is measured positioning the acetohydrazine in the same orientation as FK506-C22 ketone (Fig. 4B). In the AfFKBP12-APX879 crystal structure, two protein monomers bound to APX879 were resolved, one showing the acetohydrazine moiety in the same orientation as the FK506-C22 ketone (C21-C22 dihedral angle of $\left.68^{\circ}\right)$ and the other showing a $90^{\circ}$ rotation of the acetohydrazine moiety with a C21-C22 dihedral angle of $-44^{\circ}$ (Fig. 4C). This suggests conformational flexibility that might be restrained by calcineurin binding. Interestingly, when bound to $M c \mathrm{FKBP} 12$, APX879 acetohydrazine moiety adopts a third conformation with a $\mathrm{C} 21-\mathrm{C} 22$ dihedral angle of $-92^{\circ}$ leading to a $\sim 130^{\circ}$ flip compared to FK506C22 ketone (McFKBP12-FK506 C21-C22 dihedral angle $\sim 32^{\circ}$ ) (Fig. 4D). The adoption of this conformation prevents steric clashes with $M c$ FKBP12-Tyr88, positioned $\sim 3 \AA$ away from 
APX879-C60 when overlaid with McFKBP12-FK506. Altogether, the crystal structures suggest that the flexibility of the acetohydrazine moiety compensates, at least in part, for the bulkiness of the amino acids at position 88 . The $h$ FKBP12-His88 by itself does not prevent APX879 binding but the calcineurin complex formation might add further conformational restraints on APX879. An overlay of the A. fumigatus and bovine calcineurin-FK506-FKBP12 crystal structures (PDB $6 \mathrm{TZ7}^{20}$ and $1 \mathrm{TCO}^{30}$ ) with $h \mathrm{FKBP} 12, A f \mathrm{FKBP} 12$ and $M c \mathrm{FKBP} 12$ bound to APX879 show the conserved CnA residues Pro377 and Phe378 in the CnB binding helix (CnA-BBH) less then $3 \AA$ away from APX879 acetohydrazine moiety, supporting the necessity for a rearrangement of either the ligand or CnA-BBH for the formation of the calcineurin-APX879-FKBP12 complex.

\section{Biophysical characterization of the human versus fungal FKBP12-FK506/APX879 protein-}

\section{ligand interaction}

To identify biophysical variations between FK506 and APX879 interaction with the human and fungal FKBP12 proteins, we performed ITC assays to establish thermodynamic constants (Table 1 and Supplementary Fig. 4). As previously reported ${ }^{31-35}, h$ FKBP12 interacts with FK506 with high affinity $\left(K_{d} 2.7 \pm 0.5 \mathrm{nM}\right) . A f \mathrm{FKBP} 12$ and $M c$ FKBP12 also interact tightly with FK506 $\left(K_{d} 4.7 \pm 0.6\right.$ and $3.0 \pm 0.7 \mathrm{nM}$, respectively). The affinity for APX879 was reduced by $\sim 40$-fold for $h$ FKBP12 and McFKBP12 $\left(K_{d} \sim 120 \mathrm{nM}\right)$ and 100-fold for AfFKBP12 $\left(K_{d}=462\right.$ $\pm 23 \mathrm{nM})$. The binding stoichiometry for $h \mathrm{FKBP} 12$ and $M c$ FKBP12 was 1:1 while, for AfFKBP12, a stoichiometry of 2:1 (ligand-FKBP12) was obtained for FK506 and APX879 binding. This could be due to the displacement of a scarcely populated low-affinity homodimer or a shift in the Pro90 cis/trans conformers equilibrium triggered by FK506/APX879 binding ${ }^{19}$. All the FKBP12 proteins showed similar free energy $\left(\Delta G^{\circ}\right)$ for FK506 $(\sim-12 \mathrm{kcal} / \mathrm{mol})$ or APX879 ( -9 kcal/mol) binding. The fungal FKBP12 proteins demonstrated a $5-8 \mathrm{kcal} / \mathrm{mol}$ 
increase in enthalpy $(\Delta H)$ compared to $h \mathrm{FKBP} 12$ for FK506 binding while for APX879 they all shared a similar $\Delta H(-1.5 \mathrm{kcal} / \mathrm{mol})$. This corresponds to an increase of 6 to $13 \mathrm{kcal} / \mathrm{mol}$ for APX879 binding compared to FK506. The entropic component contribution $(T \triangle S)$ for FK506 binding is increased by $5-8 \mathrm{kcal} / \mathrm{mol}$ in the fungal FKBP12 proteins compared to $h \mathrm{FKBP} 12$. For APX879, all FKBP12 proteins showed a $T \Delta S$ of $\sim 7 \mathrm{kcal} / \mathrm{mol}$, corresponding to an 11 to 3 $\mathrm{kcal} / \mathrm{mol}$ increase compared to FK506.

NMR titrations of FK506 and APX879 into $h \mathrm{FKBP} 12$, AfFKBP12, and McFKBP12, as followed by ${ }^{1} \mathrm{H}_{-}{ }^{15} \mathrm{~N}$ HSQC, allowed for the characterization, in solution and at the residue level, of the protein responses to ligand binding (Fig. 5 and Supplementary Fig. 5). Both FK506 and APX879 induce significant chemical shift perturbations for residues Ile25, His26, $\operatorname{Thr}_{A f} / \mathrm{Cys}_{M c} 49$, Val56, Ile57, Val102, and Glu103 in the fungal FKBP12 proteins $^{29}$. Additionally, McFKBP12 Phe47, Gln48, and Glu101, and AfFKBP12 Tyr27 and Arg43 also showed significant chemical shift differences upon ligand binding. A different set of residues in $h$ FKBP12 (Val25, Ser40, Arg43, Gly52, Ile57, Glu62, Ala65, and Phe100) are observed as having large chemical shift variations between the apo and FK506/APX879 bound forms.

The chemical exchange for FK506 binding occurred in the slow exchange regime (tight binding) for all three FKBP12 proteins leading, in most cases, to the observation of the chemical shifts of both the unbound or bound populations within the intermediate titration points (Supplementary Fig. 5). Binding of APX879 to all three FKBP12 proteins produced the same response as FK506 binding. Interestingly, peak doubling at the highest ligand:FKBP12 ratios (1:1 and 2:1) was observed for residues in the $h$ FKBP12 40's loop (40-45 and Lys48), 80's loop (Thr86, Ile91, Ile92, and His95) and residues Glu62-Gly63 suggesting conformational dynamics on the NMR timescale for the bound protein. $M c$ FKBP12 also showed peak doubling for an 
increased number of residues located in the 40's, 50's and 80's loop (Ser39, Arg41, Arg43, 4850, 52-53, 56-60, 83-84, Arg86, Tyr88, Leu91, and Glu103) while none were observed in AfFKBP12. These observations suggest that APX879 binds tightly to all three FKBP12 proteins with an intermediate to slow exchange rate but $h \mathrm{FKBP} 12$ and $M c \mathrm{FKBP} 12$, when bound to APX879, experience more conformational flexibility than AfFKBP12 or when bound to FK506.

MD simulations reveal structural differences relative to the $X$-ray crystal structures and suggest conformational flexibility

To relieve crystal contacts (Supplementary table 2) that might affect the protein and ligand conformation and to substantiate conformational flexibility suggested by NMR, six $500 \mathrm{~ns}$ MD simulations were performed for each protein:ligand complex (Supplementary Fig. 6). Interactions as characterized by $\mathrm{C} \alpha-\mathrm{RMSD}$, the solvent accessible surface area, center of mass measurements, and H-bonds patterns were found to be generally similar (Supplementary Fig. 6 and Supplementary table 3). Contrastingly, the C $\alpha$-RMSF (atomic positions in the MD simulation fitted to the crystal structures) pointed to areas of the proteins and ligands exhibiting conformational differences (Supplementary Figs. 7 and 8). Both fungal proteins when bound to APX879 showed high RMSF deviations for residues 52-55 not observed for the $h$ FKBP12APX879 complex nor any of the FK506-bound counterparts suggestive of altered conformational states for these residues. The C $\alpha$-RMSF also revealed a common deviation of the 80's loop for all FKBP12:ligand complexes. The core of the FK506 and APX879 macrocycles were found to be more similar between the crystal structures and MD simulations (lower RMSF) than the solvent exposed portions of the molecules. For all FK506-bound complexes, the largest atomistic RMSF deviations occurred at atoms C40 (FK506-C21 allyl moiety) and C45 (cyclohexylidene ring C31-O-methyl) and to a lesser extent for atoms $\mathrm{C} 33, \mathrm{C} 34, \mathrm{O} 11$, and $\mathrm{O} 12$ of the 
cyclohexylidene ring which are directly involved in interactions with the 80's loop and at the interface for calcineurin binding. For complexes bound to APX879, the ligand showed significant deviations at the acetohydrazine moiety. In addition, significant deviations were observed for an extended region surrounding the cyclohexylidene ring (atoms C28-C34, C42, and $\mathrm{C} 45)$ and $\mathrm{O} 8$, and $\mathrm{O} 10$ when bound to $\mathrm{McFKBP} 12$ or $h \mathrm{FKBP} 12$. These data suggest that the crystallization process might have artificially favored the adoption of one conformer in otherwise flexible regions of the proteins and ligands.

\section{Significance of FKBP12 and FK506/APX879 contacts observed in the MD simulations}

The significance of contacts observed throughout the MD simulations was quantified allowing for identification of variations between the human and fungal complexes potentially benefiting the design of fungal-selective FK506 analogs. In all FKBP12:ligand complexes a common core of conserved residues (Tyr27, Phe37, Asp38, Phe47, Val56, Ile57, and Trp60) and one non-conserved residue $\left(\mathrm{E}_{h} / \mathrm{R}_{A f} / \mathrm{Q}_{M c} 55\right)$ made a similar number of contacts to FK506 or APX879 (Supplementary Figs. 9 and 10). In all complexes, the 80's loop residues showed a varying degree of interaction to the ligands; however, the fungal FKBP12 proteins had a significantly larger number of contacts involving this loop.

Z-score metrics allowed for the quantification of the relative significance of the contacts for the human and the fungal proteins where the average number of contacts of $A f \mathrm{FKBP} 12$ or $M c$ FKBP12 was subtracted from the average number of $h$ FKBP12 contacts (Fig. 6 and Supplementary Fig. 10). Residues Tyr83 and $\operatorname{His}_{h} / \mathrm{Phe}_{A f} / \mathrm{Tyr}_{M c} 88$ shared an increased importance for FK506 and APX879 binding to both fungal FKBP12 proteins while residues Phe49 and Ile91 were more significant for $h$ FKBP12. Interestingly, $h$ FKBP12 also relied on Gly84 and Phe100 for FK506 binding while Arg43 was notably important for APX879 binding. 
Binding to FK506 also implicated an increased number of residues (Tyr27, Val56, Trp60, and Arg86) as making differentiating contacts between the human and fungal FKBP12 proteins that are not involved in APX879 binding.

A common core of atoms of both ligands, approximating $65 \%$ of the molecules, demonstrate similar significance for binding to all three FKBP12 proteins (Fig. 6 and Supplementary Fig. 10). In the FK506-bound forms, atoms C2-C4 of the pipecolate ring and C45 (cyclohexylidene ring C31-O-methylation) are more significant for interaction with $h$ FKBP12, while C35 (pyranose ring methylation) and C37 (C19-methylation) are more significant for interaction with the fungal FKBP12 proteins. Interestingly, for the APX879 bound forms, atoms of the cyclohexylidene ring (C33, C42, and O12), pipecolate ring (C3-C4), and C15-C19 region (C15-C16, C18, C36-C37, and O8) are more significant for interaction with $h \mathrm{FKBP} 12$, while atoms $\mathrm{C} 11-\mathrm{C} 12$ and $\mathrm{C} 35$ of the pyranose ring, $\mathrm{C} 28, \mathrm{C} 30$, and $\mathrm{C} 45$ of the opposite side of the cyclohexylidene ring, and C61 of the acetohydrazine moiety show more significant interactions with the fungal FKBP12 proteins. The complexes bound to APX879 show nearly identical importance of the acetohydrazine moiety and, therefore, no significant differences are noted within this region with the exception of C61 possibly making significant interactions with the fungal FKBP12 proteins $(M c$ FKBP12 Z-score right under +1 , right above +1 for $A f \mathrm{FKBP} 12)$.

Overall these observations indicate that the fungal FKBP12 proteins rely generally on the same amino acids (most notably Tyr83 and Phe88) to interact with FK506/APX879 while $h$ FKBP12 relies significantly on the 40's loop and Ile91. The C22 acetohydrazine moiety in APX879 increases the number of significant and differentiating contacts between the human and fungal FKBP12 proteins suggesting that it might be a better starting scaffold than FK506 for 
modifications to further increase selectivity for the fungal proteins and amplify the difference in the balance between the antifungal and immunosuppressive activities.

\section{Discussion}

Targeting calcineurin is a promising approach to develop novel antifungals due to its central role in diverse cellular processes, including antifungal drug resistance and pathogenesis in the major human fungal pathogens ${ }^{3,36}$. Although FK506 efficiently targets calcineurin, currently available formulations are immunosuppressive and, therefore, cannot be used as antifungal therapeutics. Atomistic understanding of calcineurin inhibition through FK506FKBP12 binding has the potential to reveal unique features differentiating the human and fungal proteins that could be targeted to enhance fungal selectivity.

Here we report the first crystal structure of $M c$ FKBP12 bound to FK506 revealing structural similarity with other previously crystallized mammalian and fungal FKBP12 proteins (Fig. 2 and Sup. Table 1) ${ }^{19,23,24}$. Interestingly, $M c$ FKBP12 is not functionally equivalent to AfFKBP12 in its inhibitory interaction with A. fumigatus calcineurin, due to residue 88 critical charge and side-chain size requirements. MD simulations suggest that $M c \mathrm{FKBP} 12$ and McFKBP12-Y88F interact similarly with FK506, but the Tyr88Phe mutation impacts the implication of the 50's and 80's loop, central for FK506 binding. This mutation does not affect the ability of FKBP12 to bind FK506 or calcineurin but rather alters the formation of a "productive" inhibitory protein-protein interface allowing for calcineurin inhibition (Fig. 3 and

\section{Supplementary Figs. 1-3).}

Crystal structures of FKBP12 proteins bound to APX879, a less immunosuppressive FK506 analog with an acetohydrazine moiety on FK506-C22, revealed similar interactions as when bound to FK506 (Fig. 4 and Supplementary Table 1) but strikingly also revealed a high 
degree of flexibility and rearrangement of the acetohydrazine moiety preventing clashes with the FKBP12 residue 88 . This mobility might further be restricted by the formation of the ternary complex with calcineurin. In solution binding of APX879 revealed a 40-fold decrease in affinity for $h$ FKBP12 and McFKBP12 compared to FK506 while AfFKBP12 showed a 100-fold reduction in affinity (Table 1 and Supplementary Fig. 4). Consistent with APX879 being an FK506 analog, the protein responses captured by the NMR chemical shift perturbations were similar when bound to either ligands but different when comparing the human and fungal FKBP12 proteins. Interestingly, when fully bound by APX879, both $h$ FKBP12 and $M c$ FKBP12 showed several residues in the 40's, 50's and 80's loops experiencing peak doubling not observed when bound to FK506 or in AfFKBP12 (Fig. 5 and Supplementary Fig. 5). This suggests an increased conformational flexibility of regions of $h \mathrm{FKBP} 12$ and $M c$ FKBP12 central to the formation of the inhibitory ligand:protein interface, not captured solely by the crystal structures.

Areas of the proteins experiencing the greatest conformational difference between the crystal structures and the MD simulations contained a significant number of crystal contacts and are implicated in the formation of the interface for calcineurin binding (Supplementary Figs. 68, and Supplementary Table 2). The 80's loop in all complexes and the 50's loop in the fungal FKBP12 proteins, showed a high RMSF compared to the crystal structures. Though partially captured in the crystal structures, the MD simulations enabled the exploration of a broader and more detailed scope of conformational flexibility (Supplementary Fig. 7). Altogether, our MD simulation results give credence to the rationale of using conformational ensembles rather than a single X-ray-characterized structure as a search model for rational structure-based ligand $\operatorname{design}^{37-40}$. A previous report utilizing 14 targets as a test system has benchmarked that using 
$<100$ conformers to represent protein conformational flexibility versus a single conformer can increase the accuracy of the protein:ligand interaction predictions by more than $20 \%{ }^{41}$.

Comparison of the FKBP12:ligand interactions captured by the three structural biology methods used here (i.e. H-bonds in crystal structures, chemical shift perturbation by NMR, and H-bonds in MD simulations) revealed the interaction between the protein Ile57 and the ligands atom $\mathrm{O} 2$ as maintained in all complexes independently of the methodology used (Table 2 and Supplementary Table 3). Crystal structures and MD simulations show a higher degree of agreement between themselves than with the NMR titrations. This might be, in part, due to NMR data presented here being limited to the protein backbone chemical shifts $\left({ }^{15} \mathrm{~N}-\mathrm{HSQC}\right)$, where impact on the side chains is indirectly inferred, and also in part due to the MD simulations initially relying on the crystal structures. This comparison also allowed the identification of the 50's loop (residues 50, 52, 55-57, and 60) as being important in all complexes as captured at different levels by these methodologies. Interestingly, in contrast to crystal structures, both the MD simulation and NMR titrations showed the interaction between the fungal FKBP12 proteins and ligands as being similar to one another and different compared to the human FKBP12 protein.

Additionally, the MD simulations allowed for comparison of the significance of individual FKBP12:ligand interactions, and identification of regions of the ligands where modifications may contribute to enhanced fungal selectivity (Fig. 6 and Supplementary Fig. 9). Similar free energy scores $\left(\Delta \mathrm{G}^{\circ}\right)$ for the binding of FK506/APX879 to the FKBP12 proteins were obtained from the MD simulations and ITC experiments further justifying their use in rational structure based design $(\mathrm{FK} 506: \mathrm{MD} \approx-11.3 \mathrm{kcal} / \mathrm{mol}, \mathrm{ITC} \approx-11.5 \mathrm{kcal} / \mathrm{mol}$; APX879: $\mathrm{MD} \approx-10.4 \mathrm{kcal} / \mathrm{mol}, \mathrm{ITC} \approx-9 \mathrm{kcal} / \mathrm{mol})$. The 80 's loop residues Tyr83 and $\mathrm{His}_{h} / \mathrm{Phe}_{A f} / \mathrm{Tyr}_{M c} 88$ 
are key for binding to the fungal FKBP12 proteins while residues Phe49 and Ile91 are more significant for FK506/APX879 binding to $h$ FKBP12 (Fig. 6, Supplementary Fig. 10, and Table 2). $h \mathrm{FKBP} 12$ relies more significantly on the pipecolate ring $(\mathrm{C} 2-\mathrm{C} 5)$ and $\mathrm{C} 31-\mathrm{O}-$ methylation through atom C45 (cyclohexylidene ring) for FK506 binding while the C35 and C37 methylation in the vicinity of the pyranose ring are more significant for binding to the fungal FKBP12 proteins. These FK506 regions could therefore be targeted to enhance fungal FKBP12 specific binding; however, these regions are not structurally clustered and involve a small number of atoms.

The APX879 acetohydrazine moiety alters its interaction with the FKBP12 proteins in comparison to FK506 as captured by lower binding affinities, NMR binding assays, and lower MD free-energy values. Z-scores analysis revealed that atoms of the cyclohexylidene (O12, C33, and $\mathrm{C} 42)$ and pipecolate $(\mathrm{C} 3-\mathrm{C} 4)$ rings are significantly implicated for $h \mathrm{FKBP} 12$ binding, as observed for FK506, but interestingly, the C15-C19 region of APX879 is more significantly involved in binding to $h \mathrm{FKBP} 12$ (C15-C18, C36, C37, and O8). In contrast, the pyranose (C11, $\mathrm{C} 12$, and $\mathrm{C} 35)$ and part of the cyclohexylidene (C28, C30, and C45) rings show increased significance for interactions with the fungal FKBP12 proteins. In summary, although APX879 makes a greater number of structurally clustered and significant interactions with $h$ FKBP12 than with the fungal counterparts, the human and fungal FKBP12 proteins do not rely on the same atoms of APX879 for their interaction. This suggests that APX879 can serve as a beneficial scaffold for future modifications to enhance fungal selectivity.

Our findings corroborate medicinal chemistry performed on FK506 identifying the pipecolate ring as important for the immunosuppressive activity ${ }^{42}$. Our results are also concordant with a recent report on FK506 analogs modified on the pipecolate and 
cyclohexylidene (at C31) rings showing reduced immunosuppressive activity and, in some cases, maintaining antifungal activity ${ }^{43}$. Further structural and biophysical investigation of the calcineurin-APX879-FKBP12 complexes, in combination with the approaches we developed here, will greatly contribute to our understanding of the differential conformational flexibility in the human and fungal complexes, and guide the development of fungal-selective and nonimmunosuppressive FK506 analogs. 


\section{Methods}

DNA constructs, protein expression and purification. Protein expression for crystallization, isothermal titration calorimetry (ITC) and NMR were performed as previously reported ${ }^{29}$. Briefly, M. circinelloides, A. fumigatus and human FKBP12 DNA constructs in the pET-15b plasmid (expression with an N-termini hexahistidine tag (His6-tag) cleavable by thrombin) and codon-optimized for E. coli expression, were purchased from GenScript (Piscataway, NJ). Expression was performed using E. coli BL21(DE3) cells. Cells were propagated at $37^{\circ} \mathrm{C}$ with agitation to an $\mathrm{OD}_{600}$ of 0.6 in modified M9 minimal medium containing $100 \mu \mathrm{g} / \mathrm{mL}$ ampicillin (Sigma Aldrich, St. Louis, MO), $1 \mathrm{~g} / \mathrm{L} \mathrm{NH}_{4} \mathrm{Cl}$ (or ${ }^{15} \mathrm{NH}_{4} \mathrm{Cl}$ for NMR (Cambridge Isotopes, Tewksbury, MA)) and $55 \mathrm{~g} / \mathrm{L}$ of sorbitol (M. circinelloides only). Protein expression was initiated by the addition of $1 \mathrm{mM}$ Isopropyl $\beta$-d-1-thiogalactopyranoside (IPTG) and incubation for $16 \mathrm{~h}$ at $25^{\circ} \mathrm{C}$ with agitation. Cells were harvested by centrifugation at $4^{\circ} \mathrm{C}$ for 20 minutes at $6000 \times g$ and the pellet stored at $-80^{\circ} \mathrm{C}$ until purification. The cell pellets were resuspended in 30 $\mathrm{mL}$ of $50 \mathrm{mM}$ sodium phosphate, $500 \mathrm{mM} \mathrm{NaCl}, \mathrm{pH} 8.0$ buffer. Lysis was performed by three cycles of 30 seconds of sonication at a power of 12 watts with a 2-minute rest interval on ice or by French press followed by the addition of $1 \mathrm{mM}$ of phenylmethane sulfonyl fluoride (PMSF). The lysate was clarified by centrifugation $\left(4^{\circ} \mathrm{C}, 15 \mathrm{~min}\right.$ at $\left.20,000 \times g\right)$ and filtration using a 0.22 $\mu \mathrm{m}$ PES syringe filter. The chromatography was undertaken at $4^{\circ} \mathrm{C}$ using an A $\square \mathrm{KTA}$ FPLC (GE Healthcare). The clarified supernatant was loaded onto a $5 \mathrm{~mL}$ prepacked Ni-nitrilotriacetic acid (NTA) column. Protein was eluted over a 0 to $1 \mathrm{M}$ stepwise gradient of imidazole. Fractions containing FKBP12 proteins were identified by SDS-PAGE gel electrophoresis and Coomasie Blue staining. Combined fractions were dialyzed into $50 \mathrm{mM}$ sodium phosphate, $500 \mathrm{mM} \mathrm{NaCl}$, $\mathrm{pH} 8.0$ buffer to remove the imidazole $(2$ cycles in $1 \mathrm{~L}$ for 2 hours followed by 1 cycle in $2 \mathrm{~L}$ for 
16-18 h). The His6-tag was cleaved for $16 \mathrm{~h}$ at $4^{\circ} \mathrm{C}$ using $1 \mathrm{U}$ of thrombin (GE Healthcare) per $100 \mu \mathrm{g}$ of total protein. The cleaved proteins were then loaded onto the $5 \mathrm{~mL}$ prepacked Ni-NTA column to remove the cleaved His-tag and any uncleaved protein. For crystallography in presence of the ligand, APX879 or FK506 were added in a 1 to 1.5 molar ratio of FKBP12:ligand using a solution stock of the ligand at $10 \mathrm{mg} / \mathrm{mL}$ in $100 \%$ DMSO. The total volume of DMSO added was limited to less than 5\%. In all cases, protein solutions were concentrated to a volume of $\sim 2 \mathrm{~mL}$ using a 3000 MWCO Amicon concentrator. Size-exclusion chromatography using a Sephacyrl S100HR XK26/60 FPLC column was performed. Fractions containing protein were identified by SDS-PAGE gel electrophoresis and Coomasie Blue staining. Typical yields were $40 \mathrm{mg} / \mathrm{L}$ of $>98 \%$ pure protein.

Crystallization and determination of the structures of M. circinelloides bound to FK506 and M. circinelloides, A. fumigatus and H. sapiens FKBP12 bound to APX879. After size exclusion purification, the proteins were concentrated to $10 \mathrm{mg} / \mathrm{mL}$ using a $3000 \mathrm{MWCO}$ Amicon concentrator. For $M c \mathrm{FKBP} 12 / \mathrm{FK} 506$ and $h \mathrm{FKBP} 12 / \mathrm{APX} 879$, crystals were grown at $22^{\circ} \mathrm{C}$ with a hanging drop vapor diffusion setting using a 1 to 1 ratio of the protein and reservoir solutions. McFKBP12/FK506 crystals were grown in $2100 \mathrm{mM}$ DL malic acid pH 7.0. Needle shaped crystals of $h$ FKBP12/APX879 were grown with $2.5 \mathrm{M}$ ammonium sulfate, $0.1 \mathrm{M}$ sodium acetate trihydrate $\mathrm{pH} 4.6$ as the reservoir solution. For $M c$ FKBP12/APX879, the same hanging drop vapor diffusion setting was used with a protein to the reservoir solution ratio of $0.33-0.66$. Crystals were grown with $1600 \mathrm{mM}$ sodium citrate tribasic as the reservoir solution. For AfFKBP12/APX879, initial crystals were grown at $22^{\circ} \mathrm{C}$ using a hanging drop vapor diffusion setting and $10 \mathrm{mM}$ MES pH 6.0, $200 \mathrm{mM}$ zinc acetate and 15\% reagent alcohol as the reservoir solution. Protein drops were prepared using a 1 to 1 ratio of the protein and reservoir solution. A 
single crystal was then harvested to prepare a seed stock using the Hampton Research (Aliso Viejo, CA) Seed Bead kit and the Classical method. Crystals were grown in 5 mM MES pH 6.0, $200 \mathrm{mM}$ zinc acetate and $15 \%$ reagent alcohol using a 1 to 1 ratio of the protein and reservoir solution and streaking of the drop using the seed stock. All crystals were cryopreserved directly from the drop. Diffraction data were collected at the Advanced Photon Source using sector 22 BM and ID beamlines. The collected diffraction images were indexed, integrated, and scaled using HKL2000 ${ }^{44}$. Initial phases were calculated by molecular replacement using Phenix.PHASER ${ }^{45,46}$ and the PDBs 5HUA (McFKBP12/FK506 or APX879), 2PPN ( $h$ FKBP12/APX879) and 5HWB (AfFKBP12/APX879) as search models ${ }^{19,24}$. Iterative rounds of manual model building using $\operatorname{Coot}^{47}$ and automatic refinement in PHENIX ${ }^{45}$ were performed. Data collection and refinement statistics are summarized in Supplementary Table 1. The refined structures have been deposited to the Protein Data Bank (http://www.pdb.org) under the accession codes 6VRX, 6VCT, 6VCU and 6VCV.

Active-site Volume Estimation. The binding pocket cavity volume was estimated using $3 \mathrm{~V}$ :

Voss Volume Voxelator ${ }^{26}$. The estimation was made with a small sphere of $1.5 \mathrm{~A} \square$ radius and a large sphere of $7 \mathrm{~A} \square$ radius.

Isothermal titration calorimetry (ITC) experiments. After the size exclusion purification, the proteins were concentrated to $\sim 2 \mathrm{mg} / \mathrm{mL}$ using a $3000 \mathrm{MWCO}$ Amicon concentrator. Proteins were exhaustively dialyzed at $4^{\circ} \mathrm{C}$ in the ITC buffer $(50 \mathrm{mM}$ sodium phosphate, $50 \mathrm{mM}$ sodium chloride $\mathrm{pH}$ 7.0). Experiments were performed using a VP-ITC instrument (Microcal Inc. Northampton, MA) at $25^{\circ} \mathrm{C}$. All solutions were degassed under vacuum at $25^{\circ} \mathrm{C}$ for 15 minutes immediately before use. For FK506, because of its high affinity, it was used in the sample cell at a concentration of $2 \mu \mathrm{M}$ with constant stirring at $307 \mathrm{rpm}$. The protein was loaded in the syringe 
at a concentration of $25 \mu \mathrm{M}$ and titrated in by a first injection of $2 \mu \mathrm{L}$ followed by 22 injections of $6 \mu \mathrm{L}$. Following each injection, the cell was equilibrated for 3 minutes. For APX879, because of its low solubility restricting the usable concentration and its lower affinity, it was used in the syringe at a concentration of $150 \mu \mathrm{M}$. The proteins were loaded in the cell at a concentration of $10 \mu \mathrm{M}$ with constant stirring at $307 \mathrm{rpm}\left(25^{\circ} \mathrm{C}\right)$. APX879 was titrated in by a first injection of 2 $\mu \mathrm{L}$ followed by 29 injections of $8 \mu \mathrm{L}$ and equilibrated for 3 minutes. The enthalpy of binding $(\Delta \mathrm{H}, \mathrm{kcal} / \mathrm{mol})$ was determined by integration of the injection peaks minus the controls for the heat of dilution (equivalent experiments without the ligand and without the protein). The MicroCal Origin software (OriginLab Corp., Northampton, MA) was used for a variety of binding models.

Construction of the A. fumigatus strain expressing McFKBP12 and in vitro susceptibility assays. The Mcfkbp12 expression construct was codon optimized for expression in A. fumigatus and synthesized by GenScript and cloned into pUC57 vector at KpnI-BamHI sites to generate the pUC57-McFkbp12 vector. The codon optimized Mcfkbp12 gene along with the 800 bp Affkbp12 promoter from the pUC57-McFkbp12 vector was then cloned into the pUCGH ${ }^{48}$ vector at KpnIBamHI sites to generate the pUCGH-McFkbp12promo-McFkbp12 vector. To facilitate homologous recombination a 1000 bp Affkbp12 terminator was then cloned at the SbfI-HindIII on pUCGH-McFkbp12promo-McFkbp12 vector to generate the final pUCGH-McFkbp12promoMcFkbp12-McFkbp12term vector. The pUCGH-McFkbp12promo-McFkbp12-McFkbp12term vector was linearized by digestion with KpnI and the 7148 bp linearized fragment was transformed into the A. fumigatus akuB ${ }^{\mathrm{KU} 80}$ strain. Transformants were selected with hygromycin B $(150 \mu \mathrm{g} / \mathrm{ml})$. The Mcfkbp12-Y88F mutant construct was generated by site-directed mutagenesis PCR using the primers pUCGH-2033-F (GCGTTGGCCGATTCATTA) and 
McFkbp12-Y88F-R (AAGTCCAGGGAAGCCGCGCTC) to obtain a 1290 bp PCR fragment, and McFkbp12-Y88H-F (GAGCGCGGCTTCCCTGGACTT) and Hyg-R (GCCCATGAACTGGCTCTTAA) to obtain a 1267 bp PCR fragment. Fusion PCR was then performed with pUCGH-2033-F and Hyg-R using a 1:1 mixture of the two PCR fragments (1290 bp and $1267 \mathrm{bp}$ ) to obtain the final $2536 \mathrm{bp}$ PCR fragment harboring the Y88F mutation. This 2536 bp fragment was digested with NotI-KpnI and cloned into pUCGH at NotI-KpnI sites to obtain the pUCGH-McFkbp12promo-McFkbp12-Y88F vector. In the next step the Affkbp12 terminator was inserted at SbfI-HindIII as described for the pUCGH-McFkbp12promoMcFkbp12 vector followed by linearization and transformation into the A. fumigatus akuB ${ }^{\mathrm{KU} 80}$ strain. All the constructs were sequenced to confirm accuracy before using for transformations. The transformants were verified for homologous integration by PCR, and accuracy of the Mcfkbp12 and Mcfkbp12-Y88F sequences was verified by sequencing and visualized by fluorescent microscopy. E. coli DH5 $\alpha$ competent cells were used for subcloning experiments. $A$. fumigatus wild-type strain $a k u B^{K U 80}$ was used for growth and recombinant strain generation experiments. A. fumigatus wild-type or recombinant strains were cultured on glucose minimal media (GMM) or RPMI liquid media at $37^{\circ} \mathrm{C}$ for 24 or $48 \mathrm{~h}$ time periods. In certain experiments, GMM agar or RPMI liquid media were supplemented with FK506 (0.01-4 $\mu \mathrm{g} / \mathrm{mL})$ or APX879 (0.01-4 $\mu \mathrm{g} / \mathrm{mL})$. All growth experiments were repeated as technical triplicates, each also as biological triplicates.

Fluorescence microscopy. Conidia $\left(10^{4}\right)$ from the recombinant strains of A. fumigatus were inoculated into $5 \mathrm{ml} \mathrm{GMM}$ medium and poured over a sterile coverslip (22x60 mm; No.1) placed in a sterile dish $(60 \times 15 \mathrm{~mm})$. Cultures grown for $18-20 \mathrm{~h}$ at $37^{\circ} \mathrm{C}$ were observed by fluorescence microscopy using an Axioskop 2 plus microscope (Zeiss) equipped with AxioVision 4.6 imaging 
software. FK506 (100 ng/mL) was added to the cultures in order to visualize the in vivo binding of FKBP12 to the calcineurin complex at the septum.

Molecular dynamic simulations. MD simulations were performed to provide a better representation of the protein's conformational flexibility and to more accurately characterize the proteins' solution structure bound to APX879 and FK506 ${ }^{17,19}$. Crystal structures were used as the starting conformations: McFKBP12 bound to FK506 (PDB 6VRX) and APX879 (PDB 6VCT), $h \mathrm{FKBP} 12$ bound to FK506 (PDB 1FKF) and APX879 (PDB 6VCU), and AfFKBP12 bound to FK506 (PDB 5HWC - P90G mutant) and APX879 (PDB 6VCV). For the McFKBP12(Y88F) mutant bound to FK506, the wild-type crystal structure was used and mutated in silico accordingly. FK506 and APX879 small molecule parameter and topology files were downloaded and created utilizing Automated Topology Builder (ATB) and repository ${ }^{49,50}$. All molecular dynamic (MD) simulations were performed with the GROMACS 5.0.1 software package utilizing 6 CPU cores and one NVIDIA Tesla K80 GPU ${ }^{51}$. The single starting conformations used for all of the MD simulations were resulting X-ray characterized crystal structures described here or otherwise noted above. MD simulations were performed with the GROMOS54a7 force field and the flexible simple point-charge water model. The initial structures were immersed in a periodic water box with a dodecahedron shape that extended $1 \mathrm{~nm}$ beyond the protein in any dimension and neutralized with counterions. Energy minimization was accomplished through use of the steepest descent algorithm with a final maximum force below $100 \mathrm{~kJ} / \mathrm{mol} / \mathrm{min}(0.01 \mathrm{~nm}$ step size, cutoff of $1.2 \mathrm{~nm}$ for neighbor list, Coulomb interactions, and Van der Waals interactions). After energy minimization, the system was subjected to equilibration at $300 \mathrm{~K}$ and normal pressure for $1 \mathrm{~ns}$. All bonds were constrained with the LINCS algorithm (cutoff of $1.2 \mathrm{~nm}$ neighbor list, Coulomb interactions, and Van der Waals 
interactions). After temperature stabilization, pressure stabilization was obtained by utilizing the v-rescale thermostat to hold the temperature at $300 \mathrm{~K}$ and the Berendsen barostat was used to bring the system to 1 bar pressure. Production MD calculations (500 ns) were performed under the same conditions, except that the position restraints were removed, and the simulation was run for $500 \mathrm{~ns}$ (cutoff of 1.1, 0.9, and $0.9 \mathrm{~nm}$ for neighbor list, Coulomb interactions, and Van der Waals interactions). These MD simulations were repeated 6 times, with the exception of the McFKBP12(Y88F)-FK506 which was performed once. C $\alpha$-RMSD, SASA, Rg, and COM all confirmed stability and accuracy of the MD simulations by stabilizing after a $100 \mathrm{~ns}$ equilibration period (in most cases) allowing for the analysis of the last $400 \mathrm{~ns}$ of the simulations. Only three of the 36 calculations ( 6 complex repeated 6 times) were rejected from the analysis due to unstable C $\alpha$-RMSD, Rg, and/or COM (Supplementary Fig. 6).

GROMACS built-in and homemade scripts were used to analyze the MD simulation results and averaged over the 6 simulations. All images were produced using PyMOL ${ }^{51}$. Atom indices for FK506 and APX879 are provided in Supplementary Tables 4 and 5.

NMR. $\left[{ }^{15} \mathrm{~N}\right]$-labeled samples were concentrated to $0.4-0.7 \mathrm{mM}$ and buffer exchanged into 20 $\mathrm{mM}$ sodium phosphate, $100 \mathrm{mM} \mathrm{NaCl}, 0.02 \% \mathrm{NaN}_{3}$ and $5 \% \mathrm{D}_{2} \mathrm{O}, \mathrm{pH} 6.0$ using a $3000 \mathrm{MWCO}$ Amicon concentrator. All NMR experiments were performed at $25^{\circ} \mathrm{C}$, as calibrated with a standard methanol sample. Previously reported NMR backbone resonance assignments were used (BMRB Codes 27732, 27733, 27734, 27737, 27738, 27739) ${ }^{29}$. All NMR experiments were performed on Bruker Avance III spectrometer at 16.4T (700 MHz) equipped with a 4 nucleus QXI probe and pulsed-field Z-gradient. NMR data were processed using NMRPipe ${ }^{52}$ and analyzed using Sparky ${ }^{53,54}$ and NMRViewJ version $8.0^{55}$. Chemical shifts were referenced to an external 2,2-dimethyl-2-silapentane-5-sulfonate (DSS) sample. 


\section{Acknowledgement}

This work was supported by grants from the NIH/NIAID (R01 AI112595-04; P01 AI10453305). Compound APX879 was supplied by Amplyx Pharmaceuticals, Inc. Use of the Advanced Photon Source was supported by the U. S. Department of Energy, Office of Science, Office of Basic Energy Sciences, under Contract No. W-31-109-Eng-38. Data were collected at Southeast Regional Collaborative Access Team (SER-CAT) 22-ID and 22-BM beamline at the Advanced Photon Source, Argonne National Laboratory. SER-CAT is supported by its member institutions (see www.ser-cat.org/members.html), and equipment grants (S10_RR25528 and S10_RR028976) from the National Institutes of Health. The authors thank Duke's Research Computing staff for the use of Duke Computing Cluster and its support. The authors also thank Nathan Nicely and Priyamvada Acharya for the use of the equipment for protein crystallization and Michael Hoy, Zanetta Chang and Soo Chan Lee for support and discussions. Use of the Duke NMR Spectroscopy Center instrumentation is gratefully acknowledged. S.M.G. was the recipient of a Natural Sciences and Engineering Research Council of Canada (NSERC) postdoctoral fellowship.

\section{Competing interests}

The authors declare no competing interests. 


\section{References}

1. Bongomin, F., Gago, S., Oladele, R. \& Denning, D. Global and multi-national prevalence of fungal diseases-Estimate precision. Journal of Fungi 3, 57 (2017).

2. Kachroo, A.H. et al. Systematic humanization of yeast genes reveals conserved functions and genetic modularity. Science 348, 921-925 (2015).

3. Juvvadi, P.R., Lee, S.C., Heitman, J. \& Steinbach, W.J. Calcineurin in fungal virulence and drug resistance: Prospects for harnessing targeted inhibition of calcineurin for an antifungal therapeutic approach. Virulence 8, 186-197 (2017).

4. Park, H.S., Lee, S.C., Cardenas, M.E. \& Heitman, J. Calcium-calmodulin-calcineurin signaling: A globally conserved virulence cascade in eukaryotic microbial pathogens. Cell Host Microbe 26, 453-462 (2019).

5. Klee, C.B., Crouch, T.H. \& Krinks, M.H. Calcineurin: a calcium- and calmodulin-binding protein of the nervous system. Proceedings of the National Academy of Sciences of the United States of America 76, 6270-6273 (1979).

6. Hemenway, C.S. \& Heitman, J. Calcineurin. Cell Biochemistry and Biophysics 30, 115-151 (1999).

7. Clipstone, N.A. \& Crabtree, G.R. Identification of calcineurin as a key signalling enzyme in T-lymphocyte activation. Nature 357, 695-697 (1992).

8. O'Keefe, S.J., Tamura, J.i., Kincaid, R.L., Tocci, M.J. \& O'Neill, E.A. FK-506- and CsAsensitive activation of the interleukin-2 promoter by calcineurin. Nature 357, 692-694 (1992).

9. Chow, E.W.L. et al. Elucidation of the calcineurin-Crz1 stress response transcriptional network in the human fungal pathogen Cryptococcus neoformans. PLoS Genetics 13, e1006667 (2017).

10. Cramer, R.A., Jr. et al. Calcineurin target CrzA regulates conidial germination, hyphal growth, and pathogenesis of Aspergillus fumigatus. Eukaryot Cell 7, 1085-97 (2008).

11. Andreotti, A.H. Native state proline isomerization: An intrinsic molecular switch. Biochemistry 42, 9515-9524 (2003).

12. Fischer, G. \& Aumüller, T. Regulation of peptide bond cis/trans isomerization by enzyme catalysis and its implication in physiological processes. in Reviews of Physiology, Biochemistry and Pharmacology 105-150 (2003).

13. Fanghanel, J. \& Fischer, G. Insights into the catalytic mechanism of peptidyl prolyl cis/trans isomerases. Frontiers in Bioscience 9, 3453-78 (2004).

14. Aghdasi, B. et al. FKBP12, the 12-kDa FK506-binding protein, is a physiologic regulator of the cell cycle. Proceedings of the National Academy of Sciences 98, 2425-2430 (2001).

15. Harrison, R.K. \& Stein, R.L. Substrate specificities of the peptidyl prolyl cis-trans isomerase activities of cyclophilin and FK-506 binding protein: evidence for the existence of a family of distinct enzymes. Biochemistry 29, 3813-3816 (2002).

16. Schreiber, S. Chemistry and biology of the immunophilins and their immunosuppressive ligands. Science 251, 283-287 (1991).

17. Van Duyne, G., Standaert, R., Karplus, P., Schreiber, S. \& Clardy, J. Atomic structure of FKBP-FK506, an immunophilin-immunosuppressant complex. Science 252, 839-842 (1991).

18. Michnick, S., Rosen, M., Wandless, T., Karplus, M. \& Schreiber, S. Solution structure of FKBP, a rotamase enzyme and receptor for FK506 and rapamycin. Science 252, 836-839 (1991). 
19. Tonthat, N.K. et al. Structures of Pathogenic Fungal FKBP12s Reveal Possible Self-Catalysis Function. mBio 7, e00492-16 (2016).

20. Juvvadi, P.R. et al. Harnessing calcineurin-FK506-FKBP12 crystal structures from invasive fungal pathogens to develop antifungal agents. Nature Communications 10, 4275 (2019).

21. Binder, U., Maurer, E. \& Lass-Flörl, C. Mucormycosis - from the pathogens to the disease. Clinical Microbiology and Infection 20, 60-66 (2014).

22. Kohler, J.R., Casadevall, A. \& Perfect, J. The spectrum of fungi that infects humans. Cold Spring Harbor Perspectives in Medicine 5, a019273-a019273 (2014).

23. Wilson, K.P. et al. Comparative X-ray structures of the major binding protein for the immunosuppressant FK506 (tacrolimus) in unliganded form and in complex with FK506 and rapamycin. Acta Crystallographica Section D Biological Crystallography 51, 511-521 (1995).

24. Szep, S., Park, S., Boder, E.T., Van Duyne, G.D. \& Saven, J.G. Structural coupling between FKBP12 and buried water. Proteins: Structure, Function, and Bioinformatics 74, 603-611 (2009).

25. Falloon, K. et al. Characterization of the FKBP12-Encoding Genes in Aspergillus fumigatus. PLOS ONE 10, e0137869 (2015).

26. Voss, N.R. \& Gerstein, M. 3V: cavity, channel and cleft volume calculator and extractor. Nucleic Acids Res 38, W555-62 (2010).

27. Shen, Y. \& Bax, A. Prediction of Xaa-Pro peptide bond conformation from sequence and chemical shifts. Journal of Biomolecular NMR 46, 199-204 (2009).

28. Lee, Y.C. et al. NMR conformational analysis of cis and trans proline isomers in the neutrophil chemoattractant, N-acetyl-proline-glycine-proline. Biopolymers 58, 548-61 (2001).

29. Gobeil, S.M.C., Bobay, B.G., Spicer, L.D. \& Venters, R.A. 15N, 13C and 1H resonance assignments of FKBP12 proteins from the pathogenic fungi Mucor circinelloides and Aspergillus fumigatus. Biomolecular NMR Assignments 13, 207-212 (2019).

30. Griffith, J.P. et al. X-ray structure of calcineurin inhibited by the immunophilinimmunosuppressant FKBP12-FK506 complex. Cell 82, 507-22 (1995).

31. Bierer, B.E. et al. Two distinct signal transmission pathways in T lymphocytes are inhibited by complexes formed between an immunophilin and either FK506 or rapamycin. Proceedings of the National Academy of Sciences 87, 9231-9235 (1990).

32. Connelly, P.R. et al. Enthalpy of hydrogen bond formation in a protein-ligand binding reaction. Proceedings of the National Academy of Sciences 91, 1964-1968 (1994).

33. Connelly, P.R. \& Thomson, J.A. Heat capacity changes and hydrophobic interactions in the binding of FK506 and rapamycin to the FK506 binding protein. Proceedings of the National Academy of Sciences 89, 4781-4785 (1992).

34. Solomentsev, G., Diehl, C. \& Akke, M. Conformational entropy of FK506 binding to FKBP12 determined by nuclear magnetic resonance relaxation and molecular dynamics simulations. Biochemistry 57, 1451-1461 (2018).

35. Wear, M.A., Patterson, A. \& Walkinshaw, M.D. A kinetically trapped intermediate of FK506 binding protein forms in vitro: Chaperone machinery dominates protein folding in vivo. Protein Expression and Purification 51, 80-95 (2007).

36. Steinbach, W.J., Reedy, J.L., Cramer, R.A., Jr., Perfect, J.R. \& Heitman, J. Harnessing calcineurin as a novel anti-infective agent against invasive fungal infections. Nat Rev Microbiol 5, 418-30 (2007).

37. Floquet, N. et al. Normal mode analysis as a prerequisite for drug design: Application to matrix metalloproteinases inhibitors. FEBS Letters 580, 5130-5136 (2006). 
38. Antunes, D.A., Devaurs, D. \& Kavraki, L.E. Understanding the challenges of protein flexibility in drug design. Expert Opinion on Drug Discovery 10, 1301-1313 (2015).

39. Allen, S.E., Dokholyan, N.V. \& Bowers, A.A. Dynamic docking of conformationally constrained macrocycles: methods and applications. ACS Chemical Biology 11, 10-24 (2015).

40. Pallara, C., Rueda, M., Abagyan, R. \& Fernández-Recio, J. Conformational heterogeneity of unbound proteins enhances recognition in protein-protein encounters. Journal of Chemical Theory and Computation 12, 3236-3249 (2016).

41. Rueda, M., Bottegoni, G. \& Abagyan, R. Consistent improvement of cross-docking results using binding site ensembles generated with elastic network normal modes. Journal of Chemical Information and Modeling 49, 716-725 (2009).

42. Goulet, M.T., Rupprecht, K.M., Sinclair, P.J., Wyvratt, M.J. \& Parsons, W.H. The medicinal chemistry of FK-506. Perspectives in Drug Discovery and Design 2, 145-162 (1994).

43. Lee, Y. et al. In vitro and in vivo assessment of FK506 analogs as novel antifungal drug candidates. Antimicrobial Agents and Chemotherapy 62, e01627-18 (2018).

44. Otwinowski, Z. \& Minor, W. Processing of X-ray diffraction data collected in oscillation mode. in Macromolecular Crystallography Part A 307-326 (1997).

45. Adams, P.D. et al. PHENIX: a comprehensive Python-based system for macromolecular structure solution. Acta Crystallographica Section D Biological Crystallography 66, 213-221 (2010).

46. McCoy, A.J. et al. Phaser crystallographic software. Journal of Applied Crystallography 40, 658-674 (2007).

47. Emsley, P., Lohkamp, B., Scott, W.G. \& Cowtan, K. Features and development of Coot. Acta Crystallographica Section D Biological Crystallography 66, 486-501 (2010).

48. Juvvadi, P.R. et al. Calcineurin localizes to the hyphal septum in Aspergillus fumigatus: implications for septum formation and conidiophore development. Eukaryot Cell 7, 1606-10 (2008).

49. Malde, A.K. et al. An automated force field topology builder (ATB) and repository: Version 1.0. J Chem Theory Comput 7, 4026-37 (2011).

50. Stroet, M. et al. Automated topology builder version 3.0: Prediction of solvation free enthalpies in water and hexane. J Chem Theory Comput 14, 5834-5845 (2018).

51. Abraham, M.J. et al. GROMACS: High performance molecular simulations through multilevel parallelism from laptops to supercomputers. SoftwareX 1-2, 19-25 (2015).

52. Delaglio, F. et al. NMRPipe: a multidimensional spectral processing system based on UNIX pipes. J Biomol NMR 6, 277-93 (1995).

53. Goddard, T.D. \& Kneller, D.G. SPARKY 3. University of California, San Francisco, CA (2008).

54. Lee, W., Tonelli, M. \& Markley, J.L. NMRFAM-SPARKY: enhanced software for biomolecular NMR spectroscopy. Bioinformatics 31, 1325-7 (2015).

55. Johnson, B.A. \& Blevins, R.A. NMR View: A computer program for the visualization and analysis of NMR data. J Biomol NMR 4, 603-14 (1994). 


\section{Tables and Figures}

Table 1. Thermodynamic parameters for the binding of FK506 and APX879 to human, $A f$ and $M c$ FKBP12 proteins determined by ITC. Average from triplicates performed at $25^{\circ} \mathrm{C}$, See Supplementary Fig. 4 for heat pulse data.

\begin{tabular}{cccccc}
\hline \multirow{2}{*}{ FK506 } & $\begin{array}{c}K d \\
(\mathrm{nM})\end{array}$ & $\begin{array}{c}\Delta H \\
(\mathrm{kcal} / \mathrm{mol})\end{array}$ & $\begin{array}{c}T \Delta S \\
(\mathrm{kcal} / \mathrm{mol})\end{array}$ & Stoichiometry & $\begin{array}{c}\Delta G^{\circ} \\
(\mathrm{kcal} / \mathrm{mol})\end{array}$ \\
\hline$h$ FKBP12 & $2.7 \pm 0.5$ & $-15.0 \pm 1.1$ & $-3.3 \pm 1.2$ & $1.09 \pm 0.03$ & $-12.0 \pm 1.6$ \\
$A f$ FKBP12 & $4.7 \pm 0.6$ & $-6.7 \pm 0.3$ & $4.6 \pm 0.3$ & $2.02 \pm 0.06$ & $-11.3 \pm 0.4$ \\
$M c$ FKBP12 & $3.0 \pm 0.7$ & $-9.8 \pm 0.1$ & $1.9 \pm 0.2$ & $1.06 \pm 0.06$ & $-11.7 \pm 0.2$ \\
\hline \multirow{2}{*}{ APX879 } & $K d$ & $\begin{array}{c}\Delta H \\
(\mathrm{nM})\end{array}$ & $\begin{array}{c}T \Delta S \\
(\mathrm{kcal} / \mathrm{mol})\end{array}$ & $\begin{array}{c}\mathrm{kcal} / \mathrm{mol}) \\
\text { Stoichiometry }\end{array}$ & $\begin{array}{c}\Delta G^{\circ} \\
(\mathrm{kcal} / \mathrm{mol})\end{array}$ \\
\hline$h$ FKBP12 & $119.4 \pm 15.8$ & $-1.8 \pm 0.4$ & $7.4 \pm 0.8$ & $1.41 \pm 0.03$ & $-9.2 \pm 0.9$ \\
$A f$ FKBP12 & $461.6 \pm 22.6$ & $-1.2 \pm 0.4$ & $7.2 \pm 1.0$ & $1.96 \pm 0.13$ & $-8.0 \pm 1.0$ \\
$M c$ FKBP12 & $126.4 \pm 30.3$ & $-2.0 \pm 0.1$ & $7.4 \pm 0.2$ & $0.93 \pm 0.17$ & $-9.4 \pm 0.2$ \\
\hline
\end{tabular}


Table 2. Protein residues and ligand atoms implicated in significant hydrogen bond interactions between FK506 or APX879 and $h$ FKBP12, AfFKBP12, and McFKBP12 or having high $\Delta$ chemical shift while binding to FK506 or APX879. (For MD simulation Hbonds Z-scores calculation see Supplementary Table 3). Residues in blue are observed by two methods while in green are observed by the three methods for a same protein-ligand complex.

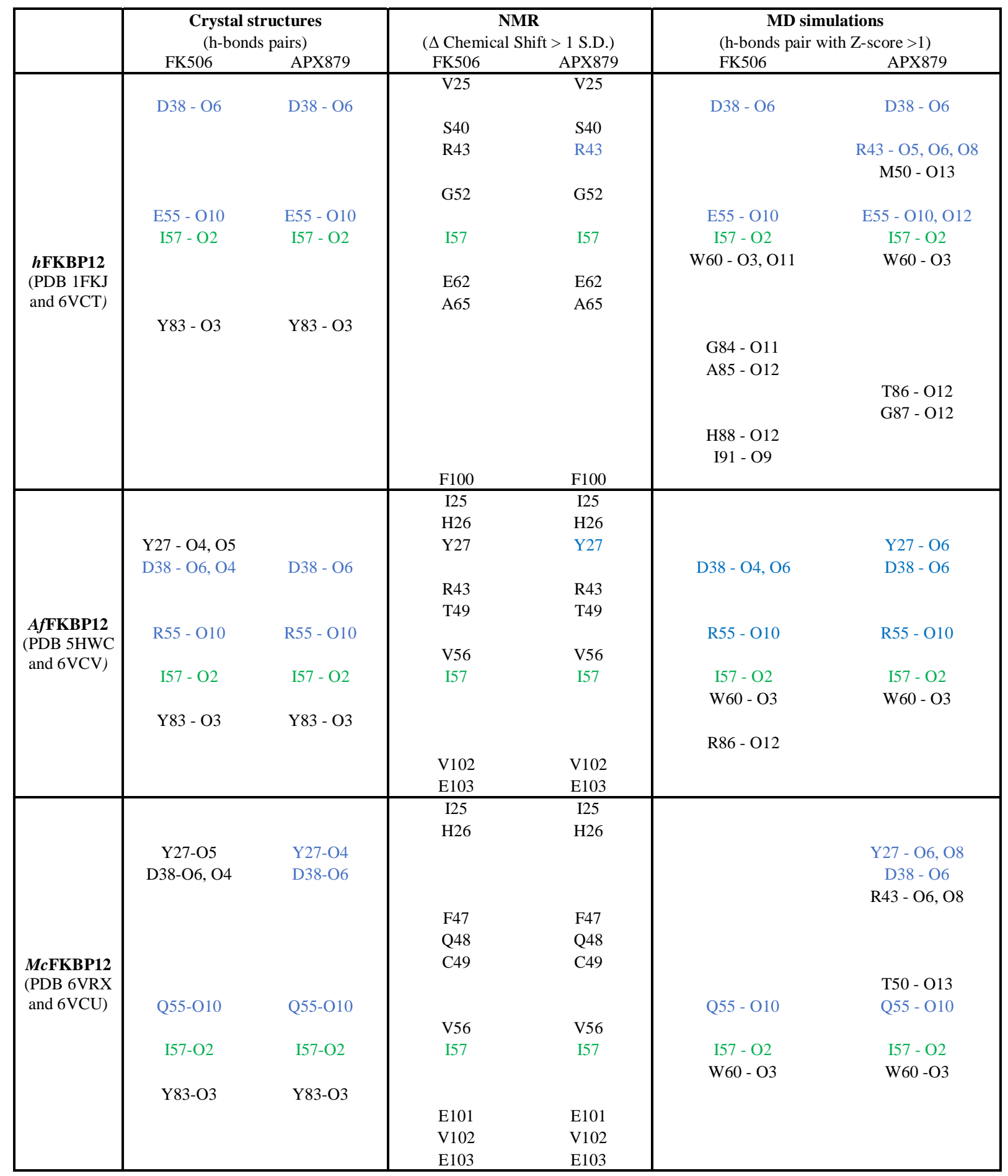


A

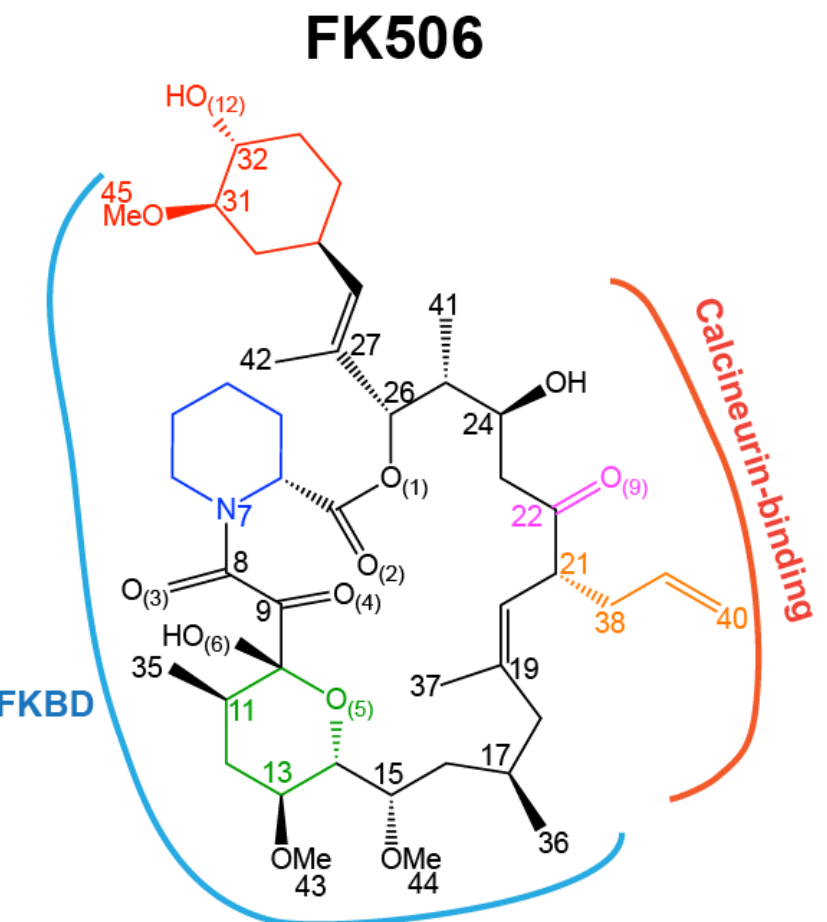

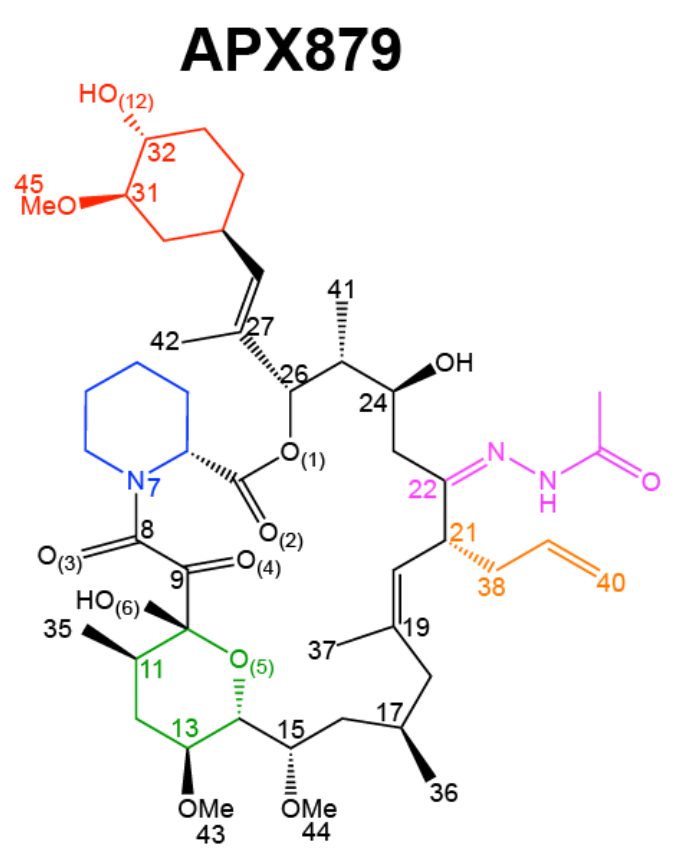

B

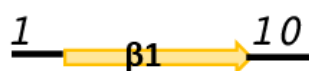

20

B2 30

40's loop

$\beta_{3} 50$ 50's loop

Hs MGVQVETISPGDGRTFPKRGQTCVVHYTGMLEDGKKFDSSRDRNKPFKFMLGKQEV

Af MGVTKELKSPGNGVDFPKKGDFVTIHYTGRLTDGSKFDSSVDRNEPFQTQIGTGRV

MC MGVTVERIAPGDGKNFPKKGDKVTIHYVGTLENGDKFDSSRDRGSPFQCTIGVGQV

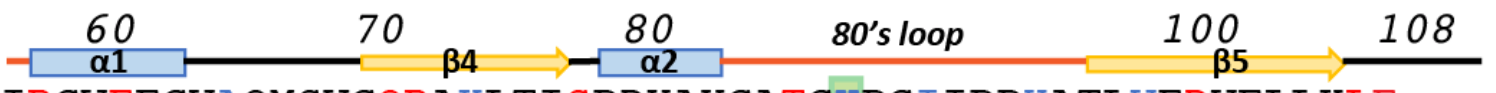

Hs IRGWEEGVAQMSVGQRAKLTISPDYAYGATGHPGI IPPHATLVFDVELLKLE----

Af IKGWDEGVPQMSLGEKAVLTITPDYGYGARGFPPVIPGNSTLIFEVELLGINNKRA

MC IKGWDEGVTQLSVGEKARLICTHDYAYGERGYPGLIPPKATLNFEVELIKIN----

Figure 1. Chemical structure of FK506 and APX879 and sequence alignment of the human,

A. fumigatus and M. circinelloides FKBP12 proteins. (A) APX879 is built on FK506 scaffold and incorporates an acetohydrazine moiety on FK506-C22. The cyclohexylidene (red), pipecolate (blue), pyranose rings (green), and the C21 allyl (orange) and C22 ketone/APX879 acetohydrazine moieties (magenta) are referenced according to FK506 atom numbering. The FKBP12 binding domain (FKBD; blue) and calcineurin binding interface (red) are indicated on FK506 (PDB 6TZ7). (B) Alignment of the human (Hs), A. fumigatus (Af), and M. circinelloides (Mc) FKBP12 proteins. Residues are colored in red when varying in one of the sequences and in blue when different in all three sequences. Residue 88 is highlighted in green. Numbering and secondary structural elements are identified. 


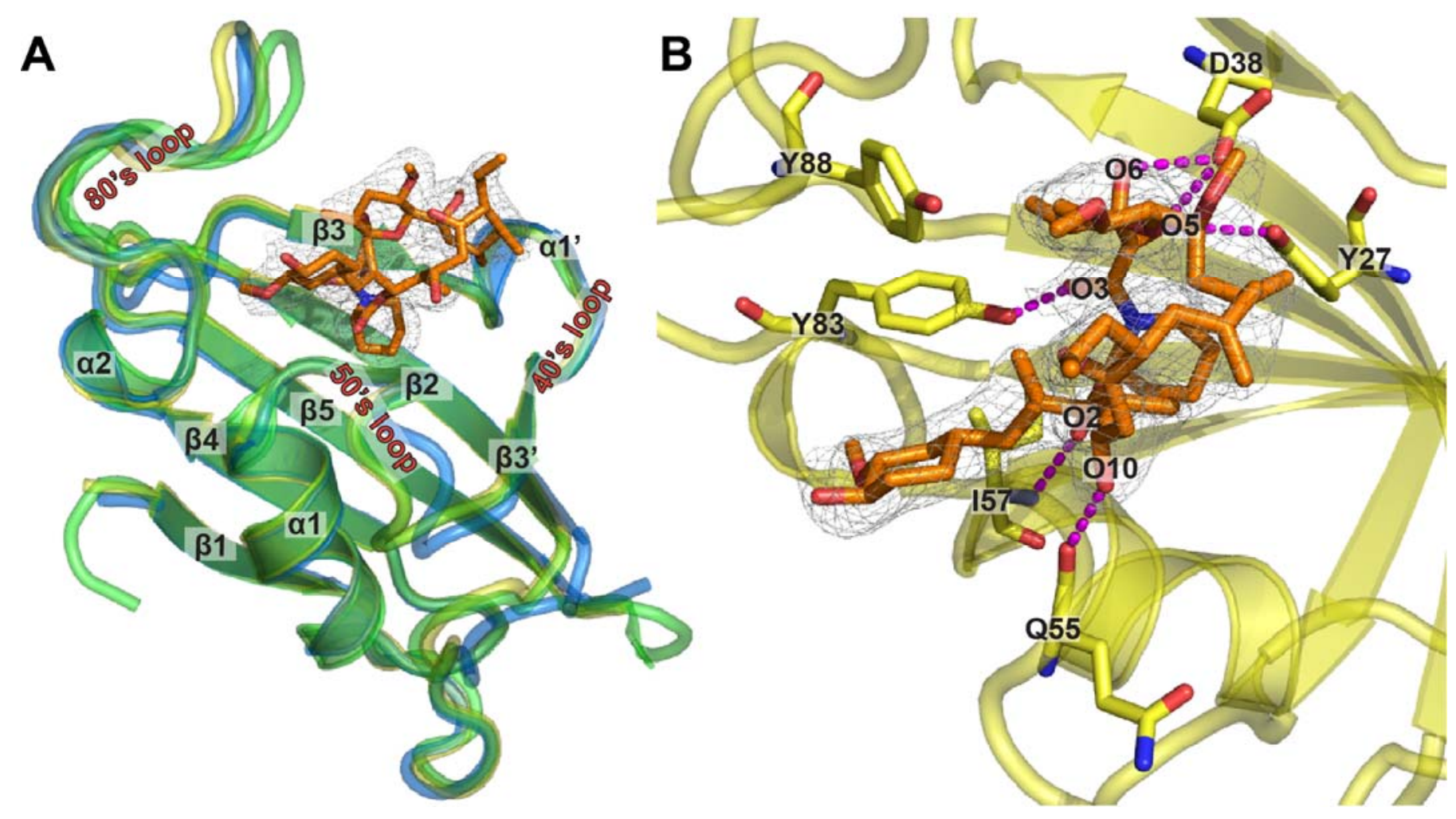

Figure 2. Crystal structure of $M$. circinelloides FKBP12 bound to FK506. (A) Overlay of the crystal structures of $M c$ FKBP12 (yellow; PDB ID 6VRX), $h$ FKBP12 (blue; PDB ID 1FKJ) and AfFKBP12-P90G (green; PDB ID 5HWC) bound to FK506. Secondary structural elements are labeled and FK506 from the McFKBP12 crystal structure is shown in orange stick representation with the $2 \mathrm{mFO}-\mathrm{dFc}$ density map at the $1 \sigma$ level. (B) FK506 binding pocket in McFKBP12. Five residues (Tyr27, Asp38, Gln55, Ile57, and Tyr83) are forming H-bonds to FK506 (in magenta dashed lines). See Supplementary Table 1 for Data collection and refinement statistics. 


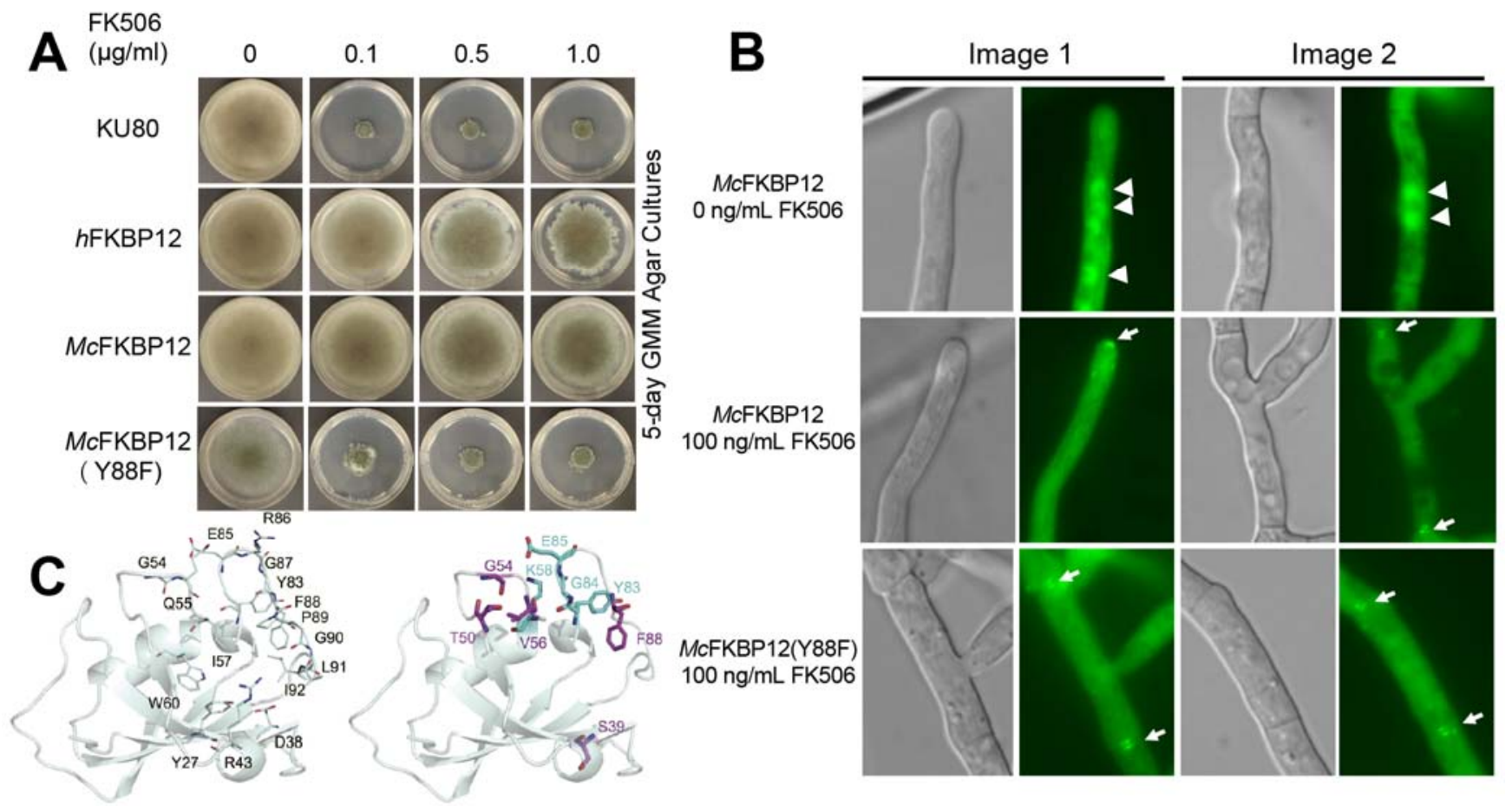

Figure 3. M. circinelloides FKBP12 does not functionally complement $A$. fumigatus

FKBP12. (A) Growth of the wild-type A. fumigatus strain (KU80) and the strain expressing the $h$ FKBP12, McFKBP12, and McFKBP12-Y88F proteins in the absence and presence of FK506 for 5-days at $37 \square$ C. (B) Microscopic localization of the McFKBP12 and McFKBP12-Y88F proteins in vivo in the absence or presence of FK506. Arrowheads show nuclear localization of $M c \mathrm{FKBP} 12$. Arrows indicate binding of $M c \mathrm{FKBP} 12$ and $M c \mathrm{FKBP} 12-\mathrm{Y} 88 \mathrm{~F}$ to $A f$ calcineurin at the hyphal septum. (C) McFKBP12(Y88F)-FK506 structure (cartoon representation) with the position of common H-bonds (line representation) noted with labeled residues (left). The right image shows the H-bonds to FK506 gained (cyan) or lost (purple) due to the Tyr88Phe mutation. 


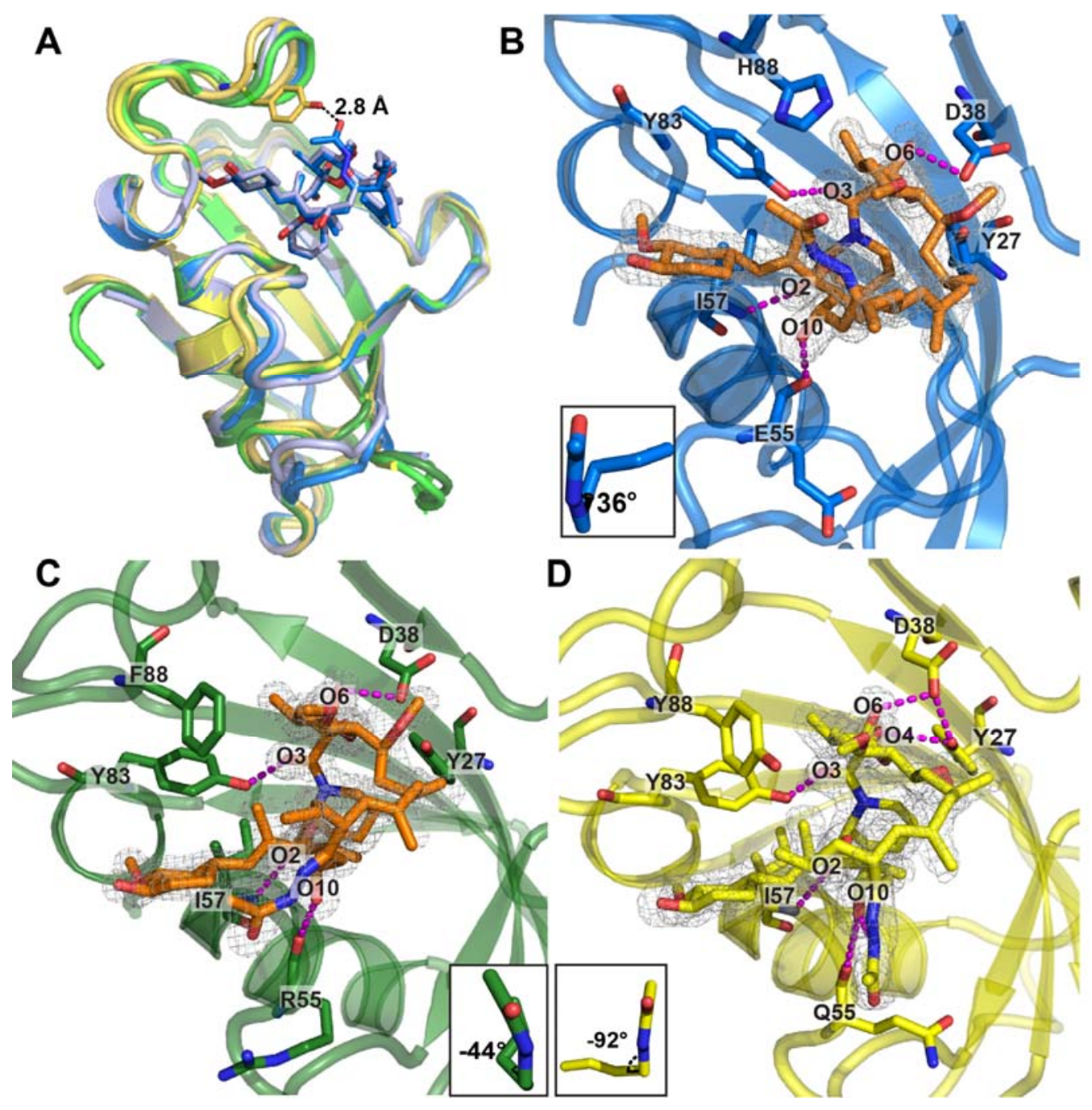

Figure 4. Crystal structures of the human, A. fumigatus, and M. circinelloides FKBP12 proteins bound to APX879. (A) Comparison of the overlaid crystal structures of McFKBP12APX879 (yellow; PDB ID 6VCT), McFKBP12-FK506 (gold; PDB ID 6VRX), hFKBP12APX879 (blue; PDB ID 6VCU), hFKBP12-FK506 (light blue; PDB ID 1FKJ), AfFKBP12APX879 (dark green; PDB ID 6VCV), and AfFKBP12(P90G)-FK506 (light green; PDB ID 5HWC). APX879 and FK506 from the $h$ FKBP12 crystal structures are represented in blue and light blue sticks. Distance to APX879-C60 and McFKBP12-Tyr88 was estimated at $2.9 \AA$. (B D) Representation of APX879 (orange) in the 2mFO-dFc density map (at $1 \sigma$ level) in the binding cavity of (B) $h \mathrm{FKBP} 12$, (C) AfFKBP12, and (D) $M c$ FKBP12. The C21-C22 dihedral angle measured are illustrated in the lower corner. Residues (Tyr27, Asp38, $\mathrm{E}_{h} / \mathrm{R}_{A f} / \mathrm{Q}_{M c} 55$, Ile57, and Tyr83) are forming H-bonds (identified by magenta dashed lines) maintaining APX879 in the binding pockets. See Supplementary Table 1 for Data collection and refinement statistics. 


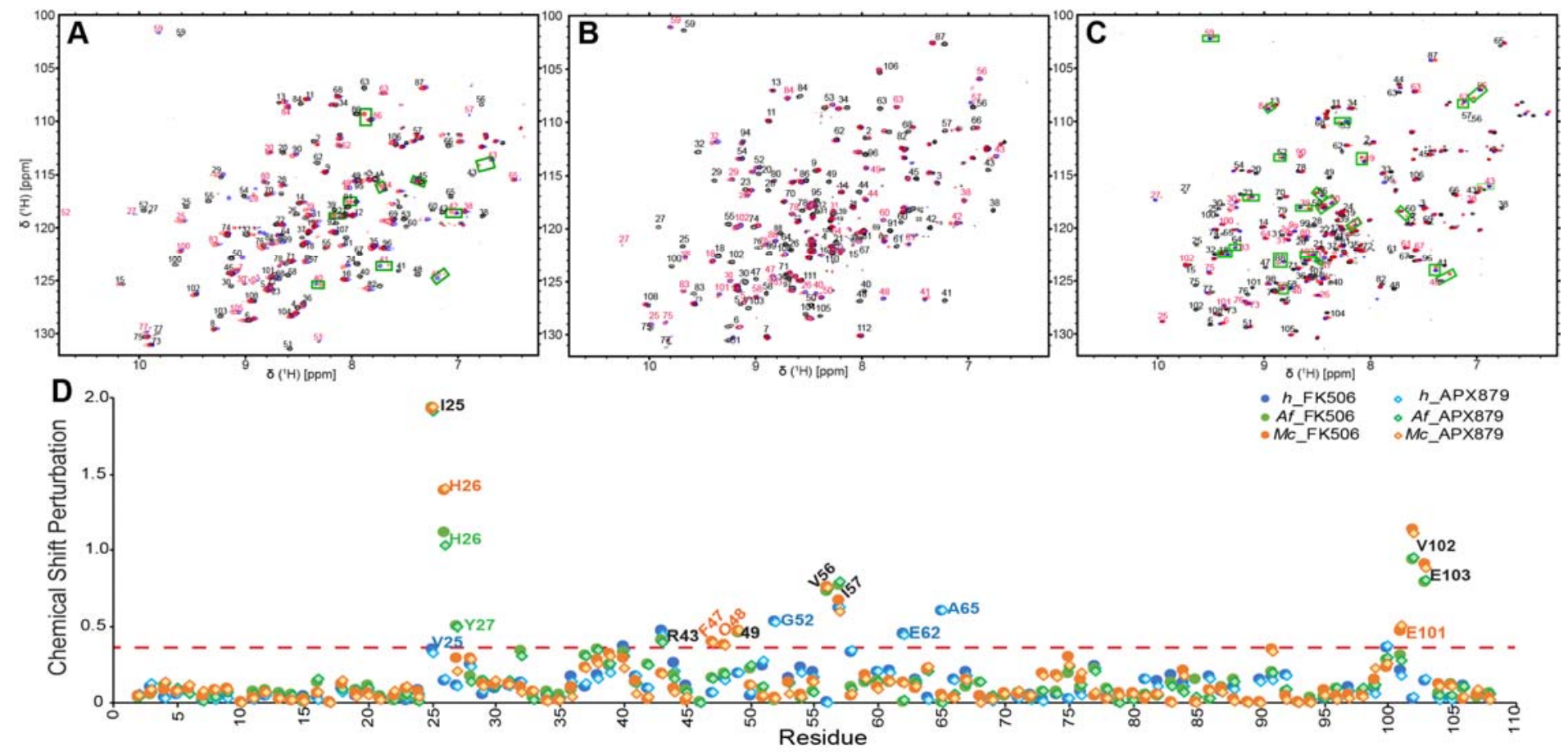

Figure 5. NMR binding of FK506 and APX879 to the human, A. fumigatus, and $M$. circinelloides FKBP12 proteins. Initial (0:1; in black) and final (2:1; in red) ${ }^{15} \mathrm{~N}-\mathrm{HSQC}$ of the titration of APX879 with the (A) human, (B) A. fumigatus and (C) M. circinelloides FKBP12 proteins. The FK506 final titration point (2:1) with the respective proteins is shown in blue for reference. Peaks doubling when protein is fully bound to APX879 are indicated by green squares (See Supplementary Fig. 5 for full titration and zoomed regions where peak doubling are observed). (D) Protein chemical shift perturbation due to FK506 and APX879 binding. The red dashed line represents significant chemical shift perturbations ( $\geq$ protein mean +1 S.D.). 
bioRxiv preprint doi: https://doi.org/10.1101/2020.04.14.039800; this version posted April 15, 2020. The copyright holder for this preprint (which was not certified by peer review) is the author/funder, who has granted bioRxiv a license to display the preprint in perpetuity. It is made available under aCC-BY-NC-ND 4.0 International license.

A
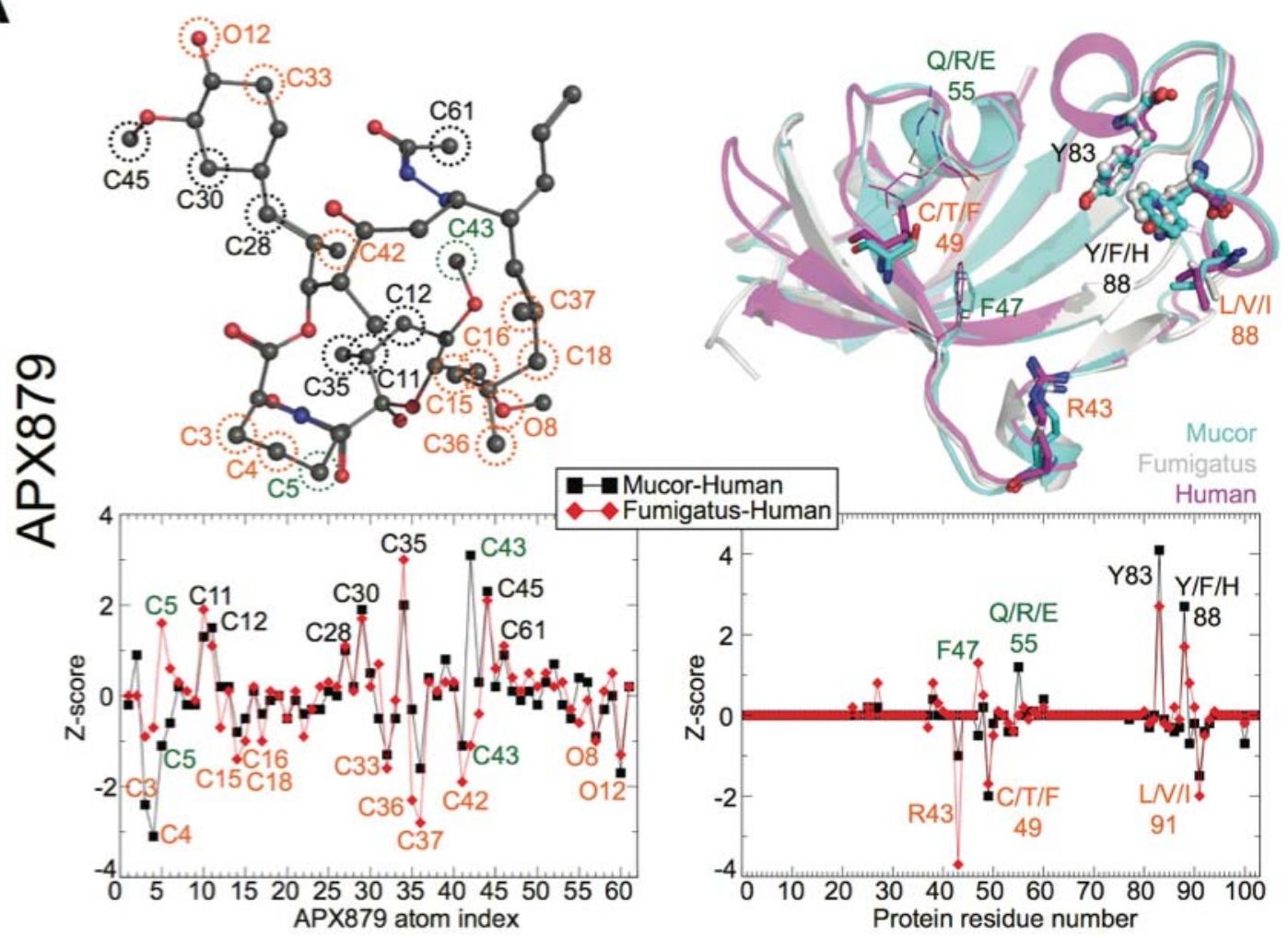

B
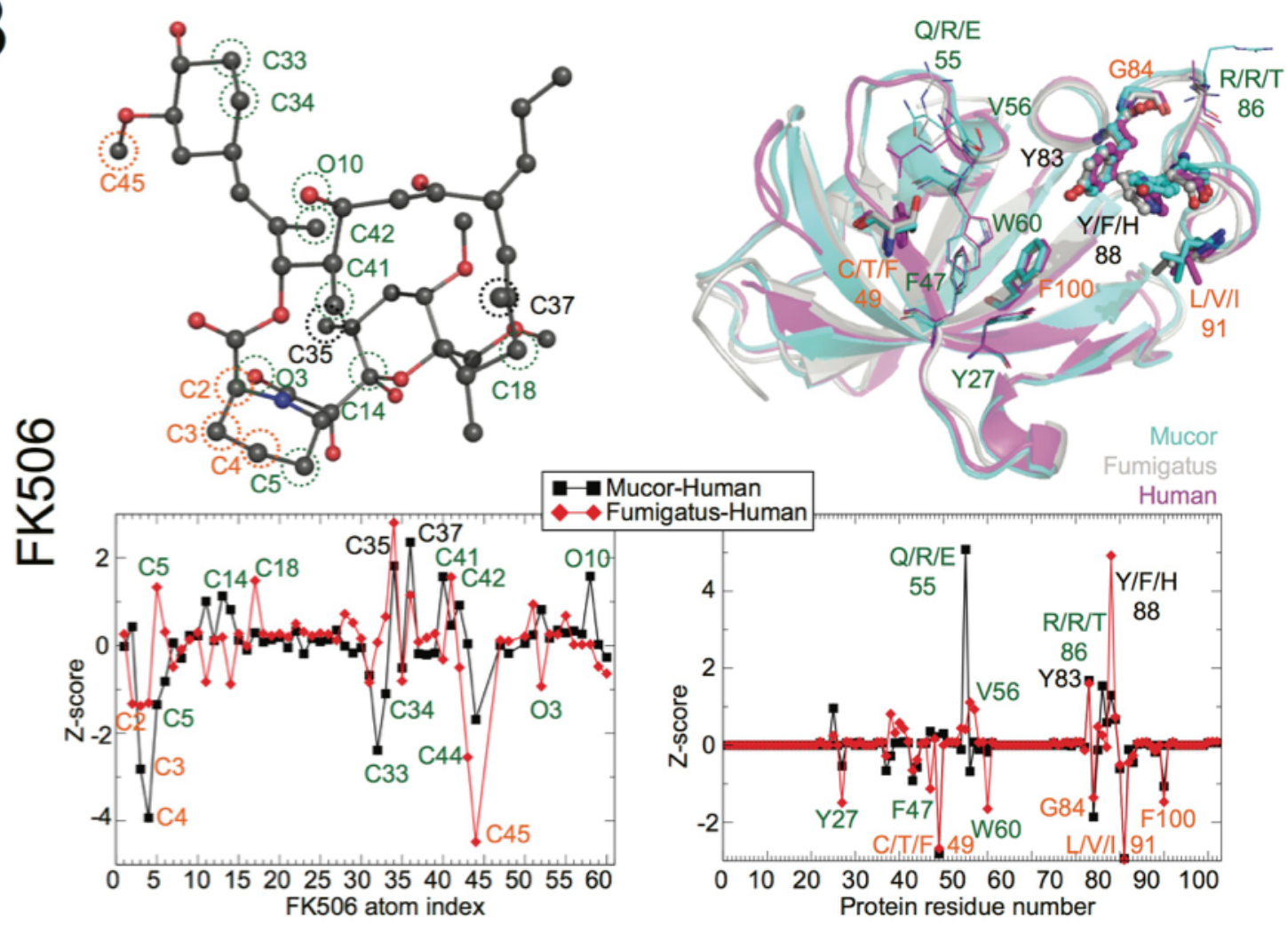
Figure 6. Ligand atoms and protein residue contacts observed in the MD simulations. Analysis of the significance of the observed contacts of $h \mathrm{FKBP} 12, A f \mathrm{FKBP} 12$, and $M c \mathrm{FKBP} 12$ bound to (A) FK506 or (B) APX879. Z-score graphs: black squares represent Z-scores of $M c \mathrm{FKBP} 12$ contacts minus $h \mathrm{FKBP} 12$ contacts, while red diamonds represent $A f \mathrm{FKBP} 12$ contacts minus $h \mathrm{FKBP} 12$ contacts. More significant contacts for the fungal proteins (Z-score >1) are labeled in black while contacts more significant for the human protein (Z-score <1) are labeled in orange. Significant contact differences between AfFKBP12 and McFKBP12 are labeled green. Left side: Stick and sphere representation of (A) FK506 and (B) APX879 colored accordingly to the atom type. Atoms making more significant contacts to $h$ FKBP12 (orange labels), the fungal proteins (black labels), and differences between the fungal AfFKBP12 and McFKBP12 (green labels) are circled using the same coloring scheme. Right side: Residues of $h$ FKBP12 (magenta), AfFKBP12 (gray), and McFKBP12 (cyan) making more significant interactions to the ligand (orange labels for $h \mathrm{FKBP} 12$, black labels for fungal proteins and green labels between the fungal AfFKBP12 and $M c$ FKBP12) are shown in stick format on the protein structures. 